\title{
Phase ordering in coupled noisy bistable systems on scale-free networks
}

\author{
Yu Atsumi \\ Department of Physics, Kyoto University, Kyoto 606-8502, Japan \\ Shigefumi Hata \\ Department of Physical Chemistry, Fritz Haber Institute of the Max Planck Society, Faradayweg 4-6, 14195 Berlin, Germany \\ Hiroya Nakao \\ Graduate School of Information Science and Engineering, Tokyo Institute of Technology, Tokyo 152-8552, Japan
}

(Received 1 August 2013; published 11 November 2013)

\begin{abstract}
We study a system consisting of diffusively coupled noisy bistable elements on a scale-free random network. This system exhibits an order-disorder phase transition as the noise intensity is varied. The phase ordering process takes place consecutively and in order of the degrees, reflecting strong degree heterogeneity of the scale-free network. A nonlinear Fokker-Planck equation describing the network dynamics is derived under mean-field approximation of the network, and is used to explain the phase ordering dynamics of the system.
\end{abstract}

DOI: 10.1103/PhysRevE.88.052806

PACS number(s): 64.60.aq, 05.45.-a

\section{INTRODUCTION}

Collective phenomena exhibited by systems consisting of interacting dynamical or stochastic elements have been the subject of significant attention over the past few decades [1-31]. One of the simplest mathematical models of such systems is an ensemble of coupled noisy bistable elements [5-8], where each individual element is described by a normal form of the pitchfork bifurcation, subjected to independent noise. If these elements are spatially distributed and diffusively coupled with their nearest neighbors, the system is equivalent to the time-dependent Ginzburg-Landau equation in the continuum limit, describing the kinetics of a nonconserved order parameter [8-10].

A system consisting of noisy bistable elements with meanfield coupling has also been studied in detail in Refs. [5-7] and has been shown to exhibit an order-disorder phase transition. In particular, Shiino [7] established the H-theorem for the nonlinear Fokker-Planck equation (NLFPE) describing the system in the continuum limit and thereby ensuring monotonous relaxation of the system into its final stationary states. Frank et al. [32,33] later extended the H-theorem to NLFPEs that describe more general systems.

In this paper, we analyze a system of noisy bistable elements that are diffusively coupled on a scale-free random network [34]. Recent studies of dynamical processes on complex networks have revealed that the topology of the network linking the elements plays a critical role on their collective behavior, as, for example, in the case of synchronization transition of nonlinear oscillators [19-22], in pattern formation in activator-inhibitor systems [23-27], and in stochastic resonance of coupled noisy bistable systems [29-31]. We generalize the previous studies for globally coupled noisy bistable systems [5-7] to network-organized systems and analyze their nontrivial phase-ordering dynamics. We show that the system exhibits an order-disorder phase transition, and the relaxation process of the system towards stationary states depends heavily on the degrees of the elements. We employ the mean-field approximation of the scale-free network to derive a set of NLFPEs approximating the network dynamics. The properties of the stationary states and the relaxation processes are argued on the basis of the approximate NLFPEs.

\section{MODEL}

\section{A. Coupled noisy bistable elements}

We consider a system consisting of noisy bistable elements on a scale-free network, in which each element is described using a scalar variable $x$, obeying a normal form of the pitchfork bifurcation, $\dot{x}(t)=x-x^{3}$. The fixed points at $x=$ \pm 1 are stable when the element is isolated, whereas the fixed point at $x=0$ is unstable. We consider $N$ such elements that are diffusively coupled through a random scale-free network and are subjected to independent noise. This system is described using coupled Langevin equations as

$$
\dot{x}_{i}(t)=x_{i}-x_{i}^{3}+\epsilon \sum_{j=1}^{N} a_{i j}\left(x_{j}-x_{i}\right)+\xi_{i}(t),
$$

for $i=1, \ldots, N$, where $x_{i}$ represents the state of the $i$ th element, $\epsilon$ is the intensity of the diffusive coupling, $\left\{a_{i j}\right\}$ is an adjacency matrix specifying the topology of the coupling network $\left(a_{i j}=1\right.$ when nodes $i$ and $j$ are connected and $a_{i j}=0$ otherwise), and $\xi_{i}(t)$ represents Gaussian white noise characterized by $\left\langle\xi_{i}(t)\right\rangle=0$ and $\left\langle\xi_{i}\left(t_{1}\right) \xi_{j}\left(t_{2}\right)\right\rangle=2 D \delta\left(t_{1}-t_{2}\right) \delta_{i j}$ with the noise intensity $D$. The degree (i.e., the number of links) of the $i$ th element is given by $k_{i}=\sum_{j=1}^{N} a_{i j}$. We generate a random scale-free network using the configuration model [35], the degree distribution of which follows the power law, $r(k) \sim k^{-\gamma}$, where $2<\gamma \leqslant 3$. The network is assumed connected, undirected $\left(a_{i j}=a_{j i}\right)$, and does not have self-loops $\left(a_{i i}=0\right)$. We fix the minimum degree at $k_{\min }=6$ and the exponent of the power law at $\gamma=2.5$ in the numerical simulations [36].

Equation (1) could be considered, for example, a simple mathematical model of opinion dynamics or consensus formation in network-organized communities of agents [37] or of a natural or artificial signaling network [29,38-40] that exhibits bistable or two-state behavior. Mathematical models similar to 
Eq. (1) have been considered by Kouvaris et al. [27] regarding traveling and stationary patterns (for the noiseless case) and in Acebrón et al. [29] and in Perc et al. [30,31], where they are used to study stochastic resonance of the network with weak periodic signals.

The coupling term in Eq. (1) describes a Laplacian diffusion on the network. If the network is globally connected $\left(a_{i j}=\right.$ $\left.1-\delta_{i j}\right)$, the coupling term is expressed as $\sum_{j=1}^{N} a_{i j}\left(x_{j}-\right.$ $\left.x_{i}\right)=N\left(\bar{x}-x_{i}\right)$ with $\bar{x}=\sum_{j=1}^{N} x_{j} / N$ denoting the mean field of the network. A system of globally coupled noisy bistable elements has been studied based on the NLFPE in the continuum limit $(N \rightarrow \infty)$ [5-7]. The system has been shown to exhibit an order-disorder phase transition, and the convergence of the system to the stationary state has been proven using the $\mathrm{H}$-theorem [7]. Our focus in this study is on the effect of the heterogeneous coupling network on the dynamics expressed by Eq. (1).

\section{B. Phase ordering process}

We first illustrate typical behavior of the system by direct numerical simulations. The initial state of the system at $t=0$ is a narrowly disturbed homogeneous state near $x=0$, i.e., each $x_{i}(0)(i=1, \ldots, N)$ is drawn independently from a sharply peaked Gaussian distribution,

$$
P_{0}(x)=\frac{1}{\sqrt{2 \pi \sigma^{2}}} \exp \left[-\frac{(x-\mu)^{2}}{2 \sigma^{2}}\right],
$$

where $\mu=0.02$ and $\sigma=0.01$. Here $\mu$ is assumed to have a slightly positive value in order to avoid complete symmetry in the initial distribution, which would lead to sensitive dependence to tiny fluctuations in the earlier stage of evolution.

Figure 1 shows snapshots of the system during the evolution process. The state of each element $x_{i}(t)$ on the network is plotted with respect to the degree $k_{i}(i=1, \ldots, N)$, which provides a convenient one-dimensional visualization of the patterns on a complex network [19-23]. The stars and squares represent the fixed points under the mean-field approximation of the network.

When the system starts evolution at $t=0$ from a slightly disturbed homogeneous state, those elements with relatively small degrees $\left(k_{i}<50\right.$ in the present case) rapidly separate into two branches, whereas those with relatively large degrees $\left(k_{i}>50\right)$ do not. The whole system therefore exhibits pitchfork-shaped patterns in the early stage of evolution $(2<t<10)$. We can explain this as follows: Equation (1) can be rewritten as

$$
\dot{x}_{i}(t)=x_{i}-x_{i}^{3}+\epsilon k_{i}\left[\bar{x}_{i}(t)-x_{i}\right]+\xi_{i}(t),
$$

where $k_{i}=\sum_{j=1}^{N} a_{i j}$ is the degree of the $i$ th element and

$$
\bar{x}_{i}(t)=\frac{1}{k_{i}} \sum_{j=1}^{N} a_{i j} x_{j}(t)
$$

is the local field experienced by the $i$ th element. At the very beginning of the evolution, all $\left|x_{i}(t)\right|$ have small values. Thus, the local field of each element is almost negligible, $\bar{x}_{i}(t) \approx 0$, and the element approximately obeys the following equation:

$$
\dot{x}_{i}(t)=\left(1-\epsilon k_{i}\right) x_{i}-x_{i}^{3}+\xi_{i}(t) .
$$

(a)

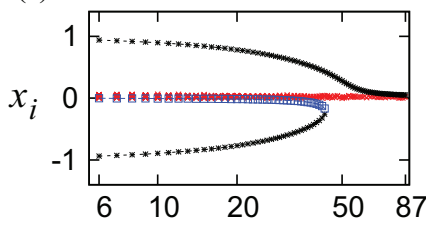

(c)

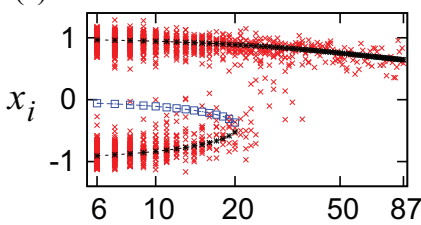

(e)

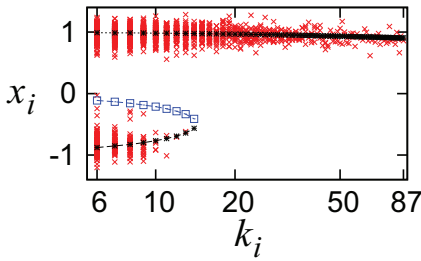

(b)

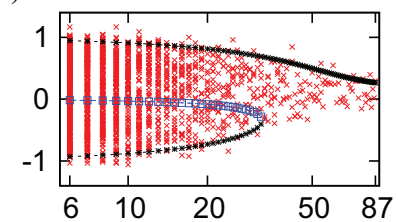

(d)

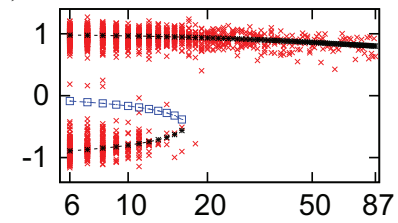

(f)

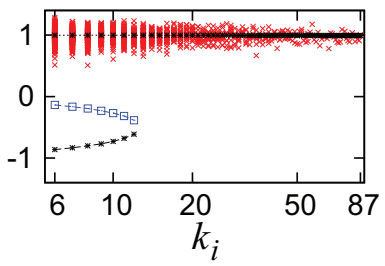

FIG. 1. (Color online) Snapshots of the distribution of the elements during numerical evolution of Eq. (1) at (a) $t=0$, (b) $t=2$, (c) $t=10$, (d) $t=100$, (e) $t=500$, and (f) $t=10000$, where the state $x_{i}$ of the element $i$ is plotted as a function of the degree $k_{i}$ for $i=1, \ldots, N$. The black stars and the blue squares represent stable and unstable fixed points of Eq. (7) under mean-field approximation, respectively, where $\bar{x}(t)$ is set to the mean field estimated using Eq. (6) from the states of the elements for each $t$. The network parameters are $N=1800, k_{\min }=6$, and $\gamma=2.5$, yielding $k_{\max }=87$. The system parameters are $\epsilon=0.02$ and $D=0.02$.

Therefore, the elements with $\epsilon k_{i}<1\left(k_{i}<50\right.$ when $\left.\epsilon=0.02\right)$ separate into two branches, becoming bimodally distributed, whereas those with $\epsilon k_{i}>1\left(k_{i}>50\right.$ when $\left.\epsilon=0.02\right)$ do not separate and retain a unimodal distribution.

As the elements with $k_{i}<50$ separate into the two branches, each local field $\bar{x}_{i}(t)$ gradually deviates from 0 , and the system starts to be distorted from its pitchfork-shaped pattern. The characteristic boundary degree $k_{\mathrm{c}}$ separating the bimodal and unimodal distributions gradually decreases due to the increase in distortion (more precisely, due to the shift in the mean field caused by the distortion; see later sections). In the case of Fig. 1, it can be seen that the unimodally distributed elements $\left(k_{i}>k_{\mathrm{c}}\right)$ as well as those elements that have separated into the upper branch $\left(k_{i}<k_{\mathrm{c}}\right)$ move rapidly upward to form an upper group $(t=100)$.

A slow phase ordering process sets in as the system evolve further $(t=500)$, and the elements that have separated into the lower branch $\left(k_{i}<k_{\mathrm{c}}\right)$ start to slowly relax. Namely, those elements in the lower branch slowly drift upward one by one to combine with the upper group. Among the separated elements belonging to the lower branch, elements with larger degrees join the upper group more rapidly, whereas those with smaller degrees take a longer time. Thus, the bimodal distribution of the elements with $k_{i}<k_{\mathrm{c}}$ that has formed in the early stage slowly transforms to a unimodal distribution centered near $x=1$ consecutively in decreasing order of the degrees.

The majority of the elements eventually join the upper group $(t=10000)$; only a small number of elements remain 
in the lower branch and only the upper group near $x=1$ survives (it is possible that elements in the lower branch completely disappear in numerical simulations using a finite number of elements). Though survival of the upper group was mostly observed in the numerical simulations because initial distributions with $\mu>0$ were used, it is possible that the lower group around $x=-1$ also survives, reflecting the symmetry of Eq. (1) with respect to $x \leftrightarrow-x$.

We here note that the parameter values used in Fig. 1, i.e., the coupling intensity $\epsilon$, the minimum degree $k_{\min }$, and the maximum degree $k_{\max }$ (which is determined by the number of elements $N$ ), are chosen to satisfy $\epsilon k_{\min }<1<\epsilon k_{\max }$, so we can observe the coexisting unimodal and bimodal distributions of the elements in the early stages of the simulation. If this condition is satisfied, coexistence of unimodal and bimodal distributions is expected at the beginning of the evolution, because Eq. (5) has a single fixed point when $\epsilon k_{i}>1$ and two fixed points when $\epsilon k_{i}<1$. If we use different parameter values, we may observe only one of the distributions from the beginning. The final stationary states can also be unimodal, bimodal, or mixed, depending on the parameters. (See Sec. IV for more details, where the final stationary states are presented for other parameter values.)

In any case, the phase ordering occurs and the approximate symmetry of the early-stage distribution is broken when the noise intensity $D$ is in an appropriate range. As we will argue in the following sections, when $D$ exceeds a certain critical value, denoted by $D_{\mathrm{c}}$, phase ordering does not occur and the symmetry of the distribution with respect to $x \leftrightarrow-x$ is recovered. The total relaxation time of the whole system depends strongly on how the elements separate into branches in the early stage of evolution. Generally, when the distribution of the elements is completely unimodal, the relaxation occurs very quickly. In contrast, when the distribution becomes completely bimodal, the relaxation time may become significantly large.

\section{MEAN-FIELD APPROXIMATION AND THE CONTINUUM LIMIT}

\section{A. Mean-field approximation}

To understand the dynamics of the system, we adopt the mean-field approximation of the scale-free network [19-23,41, 42]. It is known that the mean-field approximation works well for large random scale-free networks if the degree correlation is weak [43]. In the present case, employing the mean-field approximation is equivalent to ignoring the actual connection topology and retaining only the degrees. Namely, the local field $\bar{x}_{i}(t)$ defined in Eq. (4) is simply replaced by a degree-weighted mean field $\bar{x}(t)$ defined by

$$
\bar{x}(t)=\frac{1}{N} \sum_{j=1}^{N} \frac{k_{j}}{\bar{k}} x_{j}(t)
$$

for all $i$, where $\bar{k}=\frac{1}{N} \sum_{j=1}^{N} k_{j}$ is the mean degree of the network. Under this approximation, each set of elements that share the same degree statistically obey the same dynamics. Namely, the state variable $x$ of any element with degree $k$ is supposed to obey the following Langevin equation:

$$
\dot{x}(t)=x-x^{3}+\epsilon k[\bar{x}(t)-x]+\xi(t),
$$

for $k=k_{\min }, \ldots, k_{\max }$, where the noise $\xi(t)$ is independent for each element.

If we now assume that the value of the mean field $\bar{x}(t)$ is externally provided, Eq. (7) simply describes a single element subjected to the external field and noise. Further, if the mean field $\bar{x}(t)$ varies at a slower rate compared to the individual states of the elements [19-23], we expect that the value of the state variable $x$ will fluctuate around the stable fixed points of the deterministic part. The stars in Fig. 1 indicate the stable fixed points of Eq. (7), where $\bar{x}(t)$ is set to the mean field of the system calculated using Eq. (7) from the instantaneous distribution of elements at each instant of $t$. They are determined by $\bar{x}(t)$ and are thus time dependent. As can clearly be observed, the stable fixed points reasonably fit the backbones of the actual patterns, and the unstable fixed points act as a potential barrier that repels the elements in the later stage of the evolution, i.e., for the case where $\bar{x}(t)$ varies at a slower rate than the individual elements.

Since the elements are subjected to noise, they tend to distribute around the more stable fixed point with greater probability. We can introduce the following potential function:

$$
V(x)=V_{0}(x)+\epsilon k \frac{[x-\bar{x}(t)]^{2}}{2},
$$

where

$$
V_{0}(x)=-\frac{x^{2}}{2}+\frac{x^{4}}{4}
$$

for a given value of the mean field $\bar{x}(t)$, such that $-d V / d x=$ $x-x^{3}+\epsilon k[\bar{x}(t)-x]$ holds. In this case, the more stable fixed point, denoted here by $x_{\mathrm{g}}(k)$, corresponds to the global minimum of the above $V(x)$ in Eq. (8), likewise the less stable (metastable) fixed point, $x_{\mathrm{m}}(k)$, corresponds to the other local minimum, and the unstable fixed point, $x_{\mathrm{u}}(k)$, corresponds to the local maximum.

\section{B. Nonlinear Fokker-Planck equation}

Next we take the continuum limit $(N \rightarrow \infty)$ in order to derive a NLFPE that approximates the original network system. The mean field $\bar{x}(t)$ can be rewritten as

$$
\bar{x}(t)=\sum_{k=k_{\min }}^{k_{\max }}\left\{\frac{N(k)}{N} \frac{1}{N(k)} \sum_{j=1}^{N} \delta\left(k_{j}=k\right) \frac{k_{j}}{\bar{k}} x_{j}(t)\right\},
$$

where $\delta\left(k_{j}=k\right)$ is Kronecker's $\delta$ function and $N(k)=$ $\sum_{j=1}^{N} \delta\left(k_{j}=k\right)$ is the number of elements with degree $k$. Taking the $N \rightarrow \infty$ limit and introducing a probability density function (PDF) $P(x, t ; k)$ of the state $x$ of the element with degree $k$ at time $t$, the mean field can be expressed as

$$
\langle x\rangle(t)=\sum_{k=k_{\min }}^{k_{\max }} \frac{k}{\langle k\rangle} r(k) \int_{-\infty}^{\infty} P(x, t ; k) x d x,
$$

where $r(k)=\lim _{N \rightarrow \infty} N(k) / N$ is the degree distribution of the network and the mean degree is given by $\langle k\rangle=$ $\sum_{k=k_{\min }}^{k_{\max }} r(k) k$. We introduced here the notation $\langle\cdot\rangle$ to distinguish the continuum limit from the finite $N$ case.

Thus, in the continuum limit, i.e., in the limit of $N \rightarrow$ $\infty$ while retaining the degree distribution $r(k)$, the PDF 

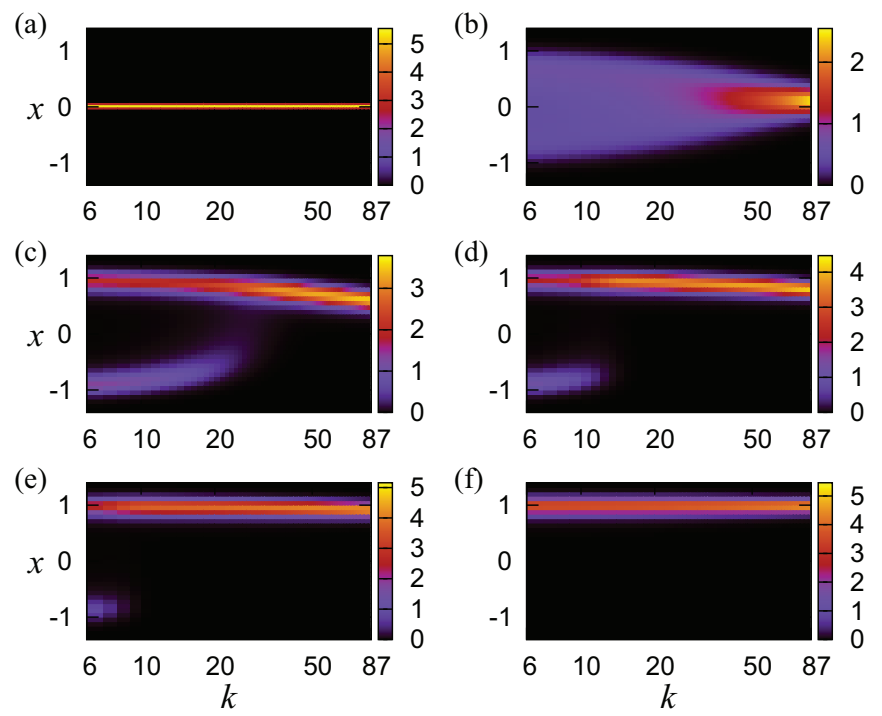

FIG. 2. (Color online) Snapshots of the probability density function $P(x, t ; k)$ for $k=k_{\min }, \ldots, k_{\max }$ during the numerical evolution of the nonlinear Fokker-Planck equation (12) at (a) $t=0$, (b) $t=2$, (c) $t=10$, (d) $t=100$, (e) $t=500$, and (f) $t=10000$, where $P(x, t ; k)$ is shown in the density plot (the bars indicate values of the probability densities). The network parameters are $k_{\min }=6$, $k_{\max }=87$, and $\gamma=2.5$, which are chosen to be the same as those used in Fig. 1, and the system parameters are $\epsilon=0.02$ and $D=0.02$.

$P(x, t ; k)$ of the elements with degree $k$ obeys the following NLFPE:

$$
\begin{aligned}
\frac{\partial P(x, t ; k)}{\partial t}= & -\frac{\partial}{\partial x}\left[\left\{x-x^{3}+\epsilon k[\langle x\rangle(t)-x]\right\} P(x, t ; k)\right] \\
& +D \frac{\partial^{2} P(x, t ; k)}{\partial x^{2}}
\end{aligned}
$$

for $k=k_{\min }, \ldots, k_{\max }$, where $\langle x\rangle(t)$ is given by Eq. (11). Note that $P(x, t ; k)$ is normalized as $\int_{-\infty}^{\infty} P(x, t ; k) d x=1$ for each $k$, and the degree distribution $r(k)$ is normalized as $\sum_{k=k_{\min }}^{k_{\max }} r(k)=1$.

\section{Evolution of the probability density functions}

Figure 2 shows typical snapshots of the PDF $P(x, t ; k)$ with respect to the degree $k$ obtained in the course of the numerical evolution of the NLFPE (12). See Fig. 1 for a comparison. The system parameters are fixed at the same values as those used in Fig. 1. The degree distribution is adjusted to that of the original network system $\left(k_{\min }=6, k_{\max }=87\right.$, and $\left.\gamma=2.5\right)$ and the system parameters $\epsilon$ and $D$ are also the same. The initial distribution of the NLFPE also corresponds to that of the network, i.e., it is represented by a sharply peaked Gaussian distribution Eq. (2) with $\mu=0.02$ and $\sigma=0.01$ for all $k=$ $k_{\min }, \ldots, k_{\max }$.

As the system starts evolution at $t=0$, the PDF $P(x, t ; k)$ of the elements with relatively small degrees (approximately satisfying $\epsilon k<1, k<50$ when $\epsilon=0.02$ ) rapidly becomes bimodal. On the other hand, the PDF for elements with relatively large degrees $(\epsilon k>1, k>50$ when $\epsilon=0.02)$ remain unimodal. The entire system therefore exhibits a distorted pitchfork-shaped pattern in the early stage $(2<t<$ 10). As it evolves, the mean field gradually deviates from zero $(\langle x\rangle \approx 0.40$ at $t=10)$ and the pitchfork-shaped pattern is further distorted, so the boundary degree $k_{\mathrm{c}}$ separating the bimodal and unimodal distributions gradually decreases. The unimodal PDF with larger $k\left(k>k_{\mathrm{c}}\right)$ and the upper branch of the bimodal PDF with smaller $k\left(k<k_{\mathrm{c}}\right)$ rapidly form an upper group near $x=1(t=100)$. The slow phase ordering process then sets in and the leftover lower branch of the PDF with $k<k_{\mathrm{c}}$ slowly relaxes into the upper group $(t=500)$. Eventually, the whole system relaxes to almost unimodal distributions around $x=1$ for all $k(t=10000)$.

Similarly to the previous case, the relaxation time largely varies depending on whether the PDF is unimodal or bimodal in the early stage of system evolution. The unimodal PDF moves up rapidly and forms the upper group near $x \approx 1$. In comparison, the bimodal PDF takes a much longer time to transform into a unimodal PDF and converges with the upper group. With respect to the bimodal PDFs for various degrees, those with relatively large degrees relax faster, whereas those with relatively smaller degrees take much longer time to converge. Thus, the relaxation process also occurs consecutively in order of the degree $k$, as in the previous simulation of the original network system.

The above results indicate that the approximate NLFPE reproduces the stationary properties and the relaxation dynamics of the original network system reasonably well.

\section{STATIONARY STATES}

\section{A. Stationary PDFs of the nonlinear Fokker-Planck equation}

We first examine stationary properties of the NLFPE (12). We assume that the mean field $\langle x\rangle(t)$ is fixed at some presumed value. The NLFPE is then decoupled into independent equations for different values of $k$, and the stationary solution for each $k$ is given by,

$$
P_{\mathrm{st}}(x ; k) \propto \exp \left\{-\frac{V_{0}(x)}{D}-\epsilon k \frac{[x-\langle x\rangle]^{2}}{2 D}\right\} .
$$

When the mean field $\langle x\rangle$ satisfies self-consistency and stability, this distribution will also be a global stationary solution for the whole NLFPE (as discussed in detail for the globally coupled case $[6,7])$.

Let $x^{(0)}$ and $x^{(1)}$ denote the presumed and resulting values of the mean field, respectively. The resulting mean field $x^{(1)}$ can be expressed as

$$
\begin{aligned}
& x^{(1)}=\sum_{k=k_{\min }}^{k=k_{\max }} \frac{k}{\langle k\rangle} r(k) x_{k}^{(1)}, \\
& x_{k}^{(1)}=\int_{-\infty}^{\infty} P_{\mathrm{st}}(x ; k) x d x,
\end{aligned}
$$

where $P_{\text {st }}(x ; k)$ is given by Eq. (13) with $\langle x\rangle=x^{(0)}$. For the self-consistency, $x^{(0)}$ should be equal to $x^{(1)}$. We denote by $x^{*}$ one of the stable self-consistent solutions to $x^{(0)}=x^{(1)}$.

Figure 3 plots $x^{(1)}$ as a function of $x^{(0)}$ for typical cases. The self-consistent solution $x^{*}$ is provided by one of the intersections of the diagonal line and the curve of $x^{(1)}$. Two nonzero stable solutions $x^{*}$ are possible when $d x^{(1)} /\left.d x^{(0)}\right|_{x^{(0)}=0}>1$ (Fig. 3, $D=0.02$ ). Similarly, when $d x^{(1)} /\left.d x^{(0)}\right|_{x^{(0)}=0}<1$, only a single stable solution exists, i.e., $x^{*}=0$ due to 


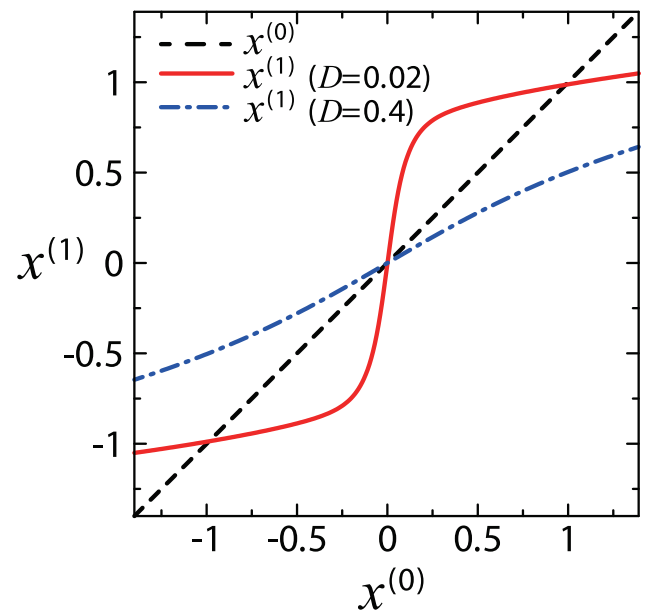

FIG. 3. (Color online) Dependence of the resulting mean field $x^{(1)}$ on the presumed mean field $x^{(0)}$ calculated from Eqs. (13) and (14) for $D=0.02<D_{\mathrm{c}}$ and $D=0.4>D_{\mathrm{c}}$. The parameter values are $k_{\min }=6, k_{\max }=87, \gamma=2.5$, and $\epsilon=0.02$.

symmetry (Fig. 3, $D=0.4$ ). It can generally be shown that $d x^{(1)} /\left.d x^{(0)}\right|_{x^{(0)}=0}<1$ as $D \rightarrow \infty$ and $d x^{(1)} /\left.d x^{(0)}\right|_{x^{(0)}=0}>1$ as $D \rightarrow+0$ [see Eqs. (A1), (A2) and (A5) in Appendix A]. Therefore, a finite critical value for $D$ (denoted by $D_{\mathrm{c}}$ ) exists at which the number of self-consistent solutions $x^{*}$ changes when $k_{\max }$ is finite, such that there exist two nonzero stable solutions if $D<D_{\mathrm{c}}$ and a single zero solution if $D>D_{\mathrm{c}}$. Namely, the noise intensity $D$ plays the role of a bifurcation parameter. The stationary PDF of the NLFPE is symmetric with respect to $x \leftrightarrow-x$ for all $k$ when $x^{*}=0$, whereas their symmetry is broken when $\left|x^{*}\right|>0$. We henceforth denote the symmetric system state (with $x^{*}=0$ ) as disordered and the asymmetric system state (with $\left|x^{*}\right|>0$ ) as ordered. From the above results, we expect that the original network system also exhibits an order-disorder transition at $D=D_{\mathrm{c}}$.

\section{B. Order-disorder transition}

We here compare the self-consistent stationary solutions $x^{*}$ of the approximate NLFPE with the mean field $\bar{x}$ of the original network system sufficiently after the initial relaxation. Figures 4(a)-4(c) show their absolute values $|\bar{x}|$ and $\left|x^{*}\right|$ (the system is statistically symmetric with respect to $x \leftrightarrow-x$ ) as functions of $D$ for $\epsilon=0.005$ (a), $\epsilon=0.02$ (b), and $\epsilon=0.2$ (c). For each figure, we obtained the residence time distribution of $\bar{x}$ using direct numerical simulations of the network in the steady state (shown in the density plot) and the self-consistent solutions of the NLFPE, $x^{*}$ (solid curves). The critical noise intensities of the NLFPE are estimated to be $D_{\mathrm{c}} \approx 0.077$ in (a), $D_{\mathrm{c}} \approx 0.184$ in (b), and $D_{\mathrm{c}} \approx 0.878$ in (c). When $D>D_{\mathrm{c}}$, the NLFPE is in the disordered phase and $x^{*}=0$. As $D$ is decreased below $D_{\mathrm{c}}$, the NLFPE enters the ordered phase and $\left|x^{*}\right|$ begins to grow. We have numerically checked that this transition is continuous and the exponent is classical, i.e., $\left|x^{*}\right| \sim\left(D_{\mathrm{c}}-D\right)^{1 / 2}$. The location of the distribution of $\bar{x}$ for the network is in reasonable agreement with the self-consistent solution $x^{*}$. Figure 4(d) illustrates the dependence of the critical noise intensity $D_{\mathrm{c}}$ on the coupling intensity $\epsilon$, where

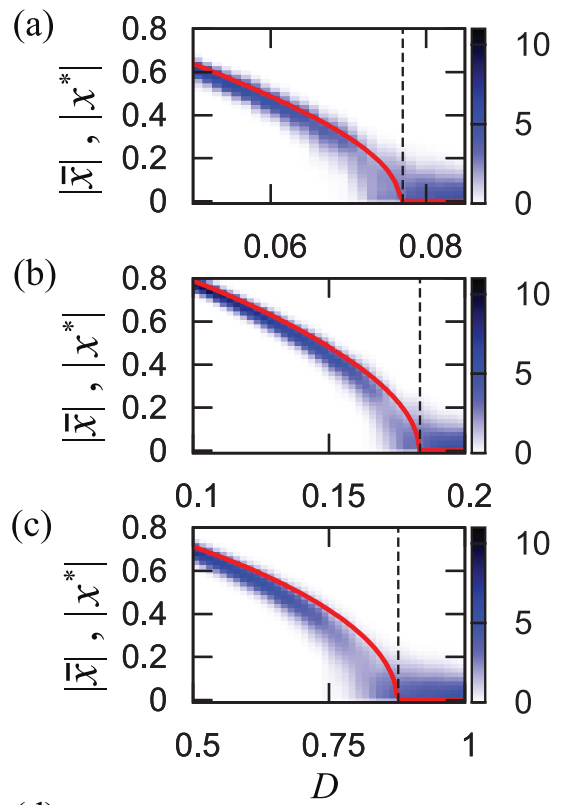

(d)

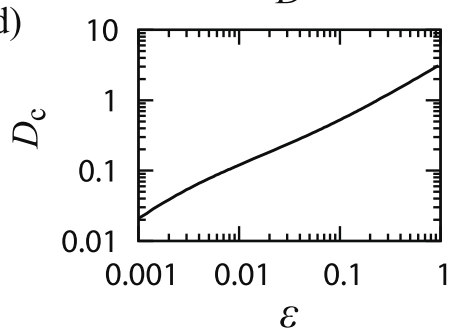

FIG. 4. (Color online) [(a)-(c)] Dependence of the mean field on the bifurcation parameter $D$ near the critical point for (a) $\epsilon=0.005$, (b) $\epsilon=0.02$, and (c) $\epsilon=0.2$. The distribution of the residence time $\tau(x)$ during which $|\bar{x}|$ stayed in the range $x \leqslant|\bar{x}|<x+\Delta x$ is depicted in the density plot (the bars indicate values of the probability densities), where $\Delta x=0.02$ and $\tau(x)$ is normalized as $\sum_{x} \tau(x)=1$. The solid lines show self-consistent solutions $x^{*}$ of the NLFPE, and the broken line indicates $D_{\mathrm{c}}$, the critical point of the NLFPE. (d) Dependence of the critical value $D_{\mathrm{c}}$ on the coupling intensity $\epsilon$. The parameters of the network are $k_{\min }=6, k_{\max }=87$, and $\gamma=2.5$.

$D_{\mathrm{c}}$ is determined using the condition $d x^{(1)} /\left.d x^{(0)}\right|_{x^{(0)}=0}=1$. The critical value $D_{\mathrm{c}}$ is generally an increasing function of $\epsilon$.

Figure 5 compares snapshots of the original network system sufficiently after initial relaxation [Figs. 5(a)-5(c)] with the stationary PDFs of the NLFPE, i.e., Eq. (13) with the mean field $\langle x\rangle$ substituted by the self-consistent solution $x^{*}$ [Figs. 5(d)-5(f)]. The parameter $\epsilon$ satisfies $\epsilon k_{\min }<1<\epsilon k_{\max }$, so the system exhibited pitchfork-shaped pattern in the early stage of evolution. Stable fixed points of Eq. (7) with $\bar{x}=x^{*}$ of the NLFPE (12) are also shown in Figs. 5(a)-5(c). We can observe that there is a reasonably good correspondence between the stationary PDFs of the NLFPE and the distributions of the elements of the original network system.

When the noise intensity $D$ is sufficiently smaller than the critical value $D_{\mathrm{c}}$, the system is deep in the ordered phase [Figs. 5(a) and 5(d)]. The upper group around $x=1$ survives in the original network model, yielding $\bar{x} \approx 1$. Correspondingly, the self-consistent solution of the NLFPE yields $x^{*} \approx 1$ and the stationary $\operatorname{PDF} P_{\mathrm{st}}(x ; k)$ is a narrowly 
(a)

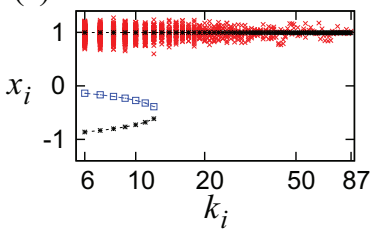

(d)

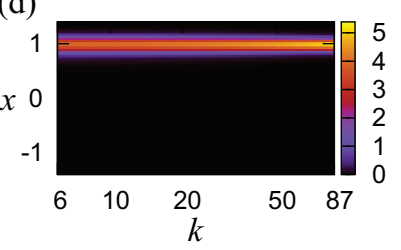

(b)

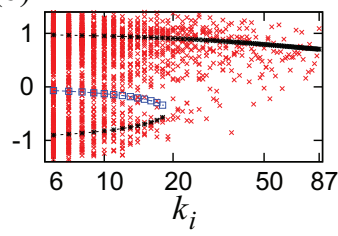

(e)

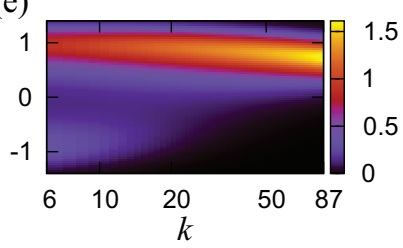

(c)

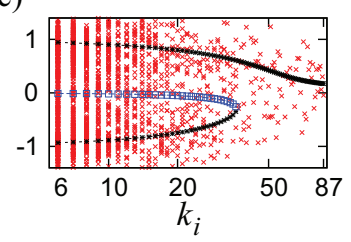

(f)

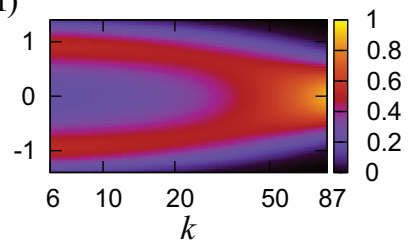

FIG. 5. (Color online) [(a)-(c)] Typical snapshots of the original network system with $N=1800$ elements sufficiently after initial relaxation obtained by direct numerical simulation of Eq. (1) at $\epsilon=0.02$ (which satisfies the condition $\epsilon k_{\min }<1<\epsilon k_{\max }$ ). The black stars and blue squares depict stable and unstable fixed points of Eq. (7), respectively, where the mean field $\bar{x}$ is estimated using Eq. (6). [(d)-(f)] Stationary probability density functions $P_{\mathrm{st}}(x ; k)$ of the nonlinear Fokker-Planck equation at $\epsilon=0.02$, shown in the density plot (the bars indicate values of the probability densities). The noise intensity is specified as $D=0.02$ in (a) and (d), $D=0.14$ in (b) and (e), and $D=0.2$ in (c) and (f). The parameters of the network are $k_{\min }=6, k_{\max }=87$, and $\gamma=2.5$.

peaked unimodal function near $x=1$ for all $k$. Regarding the stable fixed points of Eq. (7), only the upper branch remains for relatively large $k$, whereas the lower branch also persists for sufficiently small $k$. However, the stability of the persisting lower branch is very low and thus the probability density around it is invisible in the figure.

As $D$ approaches $D_{\text {c }}$, the upper group becomes more broadly distributed, especially for small $k$. Those elements with smaller degrees tend to jump between upper and lower groups more frequently on the network, so $P_{\mathrm{st}}(x ; k)$ of the corresponding NLFPE becomes wider and slightly bimodal. Since the system is in the ordered phase, the distribution of the elements is still asymmetric and, therefore, the mean field $\bar{x}$ remains nonzero.

When $D$ exceeds $D_{\text {c }}$ [Figs. 5(c) and 5(f)], the system enters the disordered phase. The distribution of the elements recovers symmetry with respect to $x \leftrightarrow-x$, and $P_{\mathrm{st}}(x ; k)$ of the NLFPE settles into a stable pitchfork pattern, which is bimodal for smaller values of $k$ and unimodal for larger values of $k$. Because of the strong noise, the distributions of the elements and the corresponding PDFs are much broader than those in the ordered phase.

Figure 6 shows the other cases with $\epsilon k_{\max }<1$ or $\epsilon k_{\min }>1$, where typical snapshots of the original network system sufficiently after the initial relaxation and the stationary PDFs of the NLFPE are compared. When $\epsilon k_{\max }<1$ [Figs. 6(a)-6(c)], the early-stage distribution is bimodal for all $k$. As the system relaxes, the distribution becomes unimodal ( $D \ll D_{\text {c }}$ ), partly bimodal ( $D$ close to $D_{\mathrm{c}}$ ), or completely bimodal for all $k\left(D>D_{\mathrm{c}}\right)$. In contrast, when $\epsilon k_{\min }>1$ [Figs. 6(d)-6(f)], the early-stage distribution is unimodal for all $k$ from the beginning and quickly relaxes either to a narrow unimodal distribution near $x=1\left(D \ll D_{\mathrm{c}}\right)$ to a slightly broader unimodal distribution in $0<x<1$ ( $D$ close to $D_{\mathrm{c}}$ ) or to a broad unimodal distribution around $x=0\left(D>D_{\mathrm{c}}\right)$, depending on the parameters. In any case, a symmetrybreaking order-disorder phase transition occurs at $D=D_{\text {c }}$ in the original network system as well as in the approximate NLFPE. The results of both systems agree reasonably.

\section{H-theorem for the nonlinear Fokker-Planck equation}

It can also be shown that the H-theorem holds for the NLFPE (12), which ensures monotonous convergence of the NLFPE to a stable stationary solution. We can define a Lyapunov functional $I$ of the PDF $P(x, t ; k)$ for $k=k_{\min }, \ldots, k_{\max }$ as

$$
\begin{aligned}
I= & \sum_{\ell} r(\ell) \int P(x, t ; \ell)\left[\ln P(x, t ; \ell)+\frac{V_{0}(x)}{D}\right] d x \\
& +\frac{\epsilon}{2 D\langle k\rangle} \sum_{\ell} \ell r(\ell) \sum_{m} m r(m) \\
& \times \iint P(x, t ; \ell) P(y, t ; m) \frac{(x-y)^{2}}{2} d x d y,
\end{aligned}
$$

which decreases monotonously with the evolution of the NLFPE [7,32] and is minimized when $P(x, t ; k)$ reaches the self-consistent solution for all $k$. See Appendix B for a discussion of the Lyapunov functional $I$.

\section{RELAXATION PROCESS}

\section{A. Consecutive phase ordering}

We here focus on the phase ordering process of the system, assuming that the noise intensity $D$ is sufficiently smaller than the critical value $D_{\mathrm{c}}$. We also assume that the diffusion constant $\epsilon$ satisfies $\epsilon k_{\min }<1<\epsilon k_{\max }$, which leads to formation of the pitchfork-shaped distribution in the early stage of system evolution. The unimodally distributed elements (with relatively large $k$ ) along with the upper branch of the bimodally distributed elements (with relatively small $k$ ) quickly form the upper group. Slow relaxation of the elements into the upper group, which are initially in the lower branch of the bimodal distribution, occurs consecutively and in order of their degrees. 
(a)
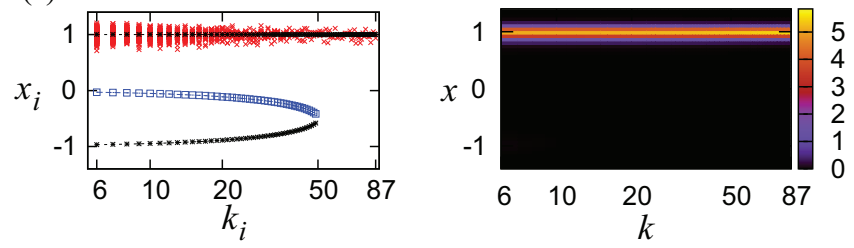

(b)
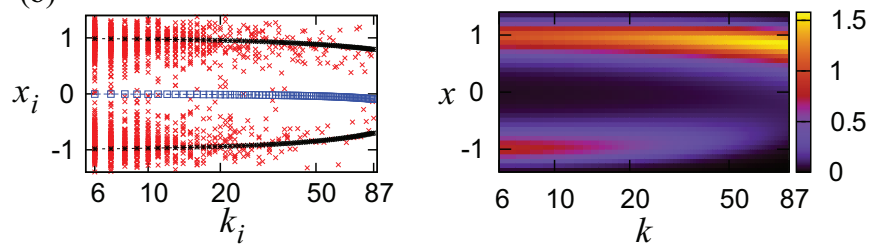

(c)
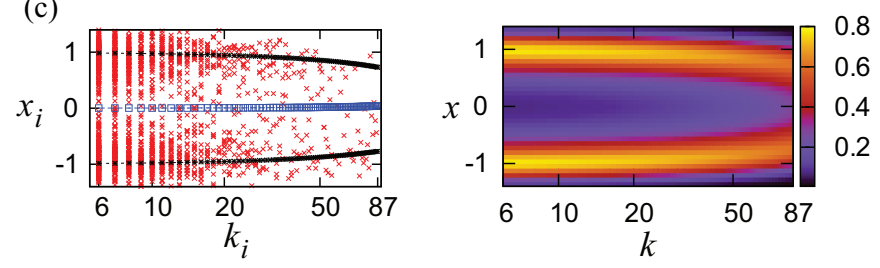

(d)
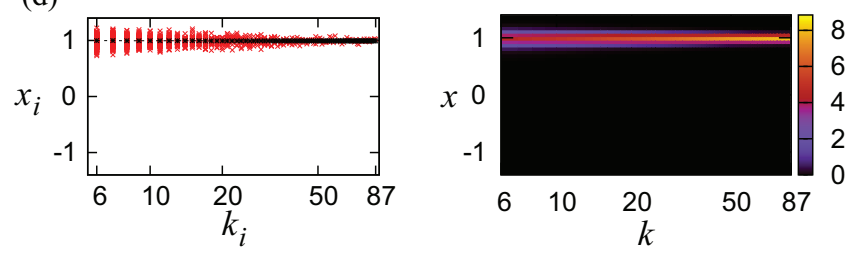

(e)
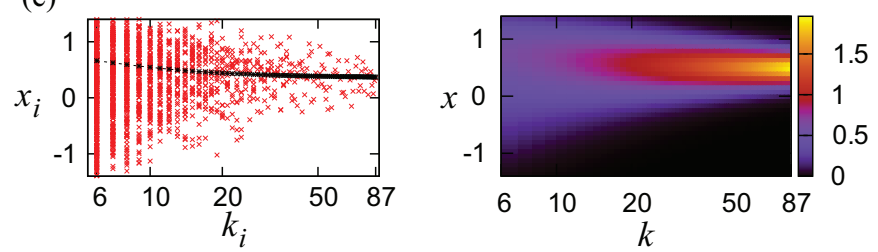

(f)
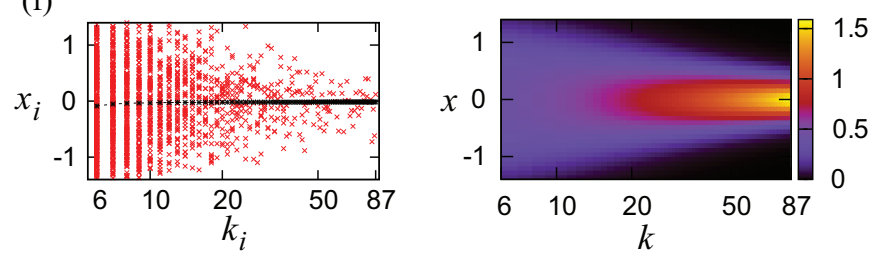

FIG. 6. (Color online) Typical snapshots of the original network system with $N=1800$ elements sufficiently after initial relaxation (left column) and the stationary probability density functions of the nonlinear Fokker-Planck equation (right column, shown in the density plot where the bars indicate values of the probability densities). The black stars and blue squares in left column depict stable and unstable fixed points of Eq. (7), respectively, where the mean field $\bar{x}$ is estimated using Eq. (6). [(a)-(c)] $\epsilon=0.005$ (satisfying $\epsilon k_{\max }<1$ ) with (a) $D=0.01$, (b) $D=0.07$, and (c) $D=0.1$. [(d)-(f)] $\epsilon=0.2$ (satisfying $\epsilon k_{\min }>1$ ) with (d) $D=0.02$, (e) $D=0.7$, and (f) $D=1$. The parameters of the network are $k_{\min }=6, k_{\max }=87$, and $\gamma=2.5$.

The consecutive relaxation process can be visualized by plotting the evolution of $\bar{x}_{k}$, i.e., the mean field of the elements
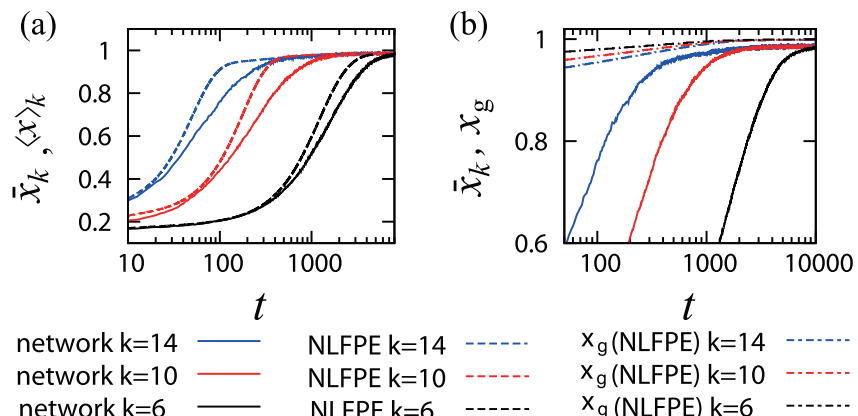
network $\mathrm{k}=6 \quad$ NLFPE $\mathrm{k}=6 \quad$-.-.- $\quad \mathrm{x}_{\mathrm{g}}$ (NLFPE) $\mathrm{k}=6 \quad$-......

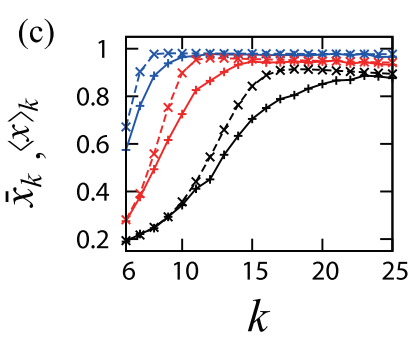

network $\mathrm{t}=1200$ network $\mathrm{t}=300$ network $\mathrm{t}=60$

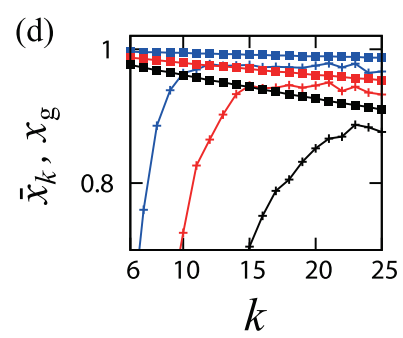

(d)

NLFPE $\mathrm{t}=1200--*--$ NLFPE $t=300-\cdots--\quad x_{g}$ (NLFPE) $t=300-$ NLFPE $t=60 \quad--*--\quad x_{g}$ (NLFPE) $t=60 \quad \longrightarrow$
FIG. 7. (Color online) (a) Evolution of $\bar{x}_{k}$ of the elements with degree $k$ of the original network system $(N=1800)$ and $\langle x\rangle_{k}$ of the approximate NLFPE for several values of $k$. (b) Evolution of $\bar{x}_{k}$ and the stable fixed point $x_{\mathrm{g}}(k)$ minimizing Eq. (8) for several values of $k$, where $x_{\mathrm{g}}(k)$ is calculated by using the mean field $\bar{x}=\langle x\rangle$ obtained from the NLFPE. (c) Snapshots of $\bar{x}_{k}$ and $\langle x\rangle_{k}$ plotted as functions of $k$ at several values of $t$. (d) Snapshots of $\bar{x}_{k}$ and $x_{\mathrm{g}}(k)$ plotted as functions of $k$ for several values of $t$. The system parameters are $N=1800, k_{\min }=6, k_{\max }=87, \gamma=2.5, D=0.02$, and $\epsilon=0.02$. The mean field of the original network system is averaged over 50 realizations.

with degree $k$. For the original network system, $\bar{x}_{k}$ is defined as

$$
\bar{x}_{k}(t)=\frac{1}{N(k)} \sum_{j=1}^{N} \delta\left(k_{j}=k\right) x_{j}(t),
$$

and for the approximate NLFPE, it can be calculated from $P(x, t ; k)$ as

$$
\langle x\rangle_{k}(t)=\int_{-\infty}^{\infty} P(x, t ; k) x d x .
$$

Figure 7(a) depicts the time evolution of $\bar{x}_{k}(t)$ and $\langle x\rangle_{k}(t)$ for several values of $k$, and Fig. 7(c) displays snapshots of $\bar{x}_{k}(t)$ and $\langle x\rangle_{k}(t)$ as functions of $k$ during the evolution. The values of $\bar{x}_{k}(t)$ and $\langle x\rangle_{k}(t)$ are in qualitative agreement, though $\langle x\rangle_{k}(t)$ tends to relax faster than $\bar{x}_{k}(t)$. From Figs. 7(a) and 7(c), we can clearly observe that the relaxation process takes place consecutively and in order of the degrees, and those elements with larger degrees relax at a faster rate.

The late stage of evolution is shown in Figs. 7(b) and 7(d), where each $\bar{x}_{k}(t)$ or $\langle x\rangle_{k}(t)$ approaches the global minimum $x_{\mathrm{g}}(k)$ of $V(x)$ in Eq. (8) with $\bar{x}(t)=\langle x\rangle(t)$ consecutively from the elements with larger $k$ [note that $x_{\mathrm{g}}(k)$ still depends on time during relaxation]. The value of $\bar{x}_{k}(t)$ or $\langle x\rangle_{k}(t)$ then follows the evolution of $x_{\mathrm{g}}(k)$ as $\bar{x}(t)$ relaxes to the final stationary 
value $x_{\mathrm{g}}^{*}(k)$, which minimizes $V(x)$ when $\bar{x}(t)$ is equal to the self-consistent solution $x^{*}$.

\section{B. Escape rate argument}

To understand the dependence of the relaxation process on the degrees of the elements, we here adopt the Kramers' escape rate theory [44-47]. Namely, we focus on the group of elements with relatively small degree $k$, which is bimodally distributed in the early stage around the two stable fixed points.

We assume that each element escapes from a metastable state (i.e., a less stable fixed point) $x_{\mathrm{m}}(k)$ to a stable state (more stable fixed point) $x_{\mathrm{g}}(k)$ over the peak of a potential barrier between them. We also assume that each element goes over the barrier individually at a low rate, so the mean field $\langle x\rangle(t)$ is maintained at an almost constant level when a single element crosses the barrier, as actually observed in numerical simulations.

Suppose that a particle driven by Gaussian white noise escapes over a potential barrier of height $V_{\mathrm{u}}$. The Kramers' theory tells that the escape time $T$ scales as $T \propto \exp \left(V_{\mathrm{u}} / D\right)$. In the present case, the potential function $V(x)$ is given by Eq. (8), and the potential barrier $V_{\mathrm{u}}$ is expressed as the difference in the potential $V(x)$ between the unstable fixed point at $x_{\mathrm{u}}(k)$ and the less stable fixed point at $x_{\mathrm{m}}(k)$,

$$
\begin{aligned}
V_{\mathrm{u}}(\langle x\rangle ; k)= & V\left(x_{\mathrm{u}}(k)\right)-V\left(x_{\mathrm{m}}(k)\right) \\
= & \left\{V_{0}\left(x_{\mathrm{u}}(k)\right)+\epsilon k \frac{\left[x_{\mathrm{u}}(k)-\langle x\rangle\right]^{2}}{2}\right\} \\
& -\left\{V_{0}\left(x_{\mathrm{m}}(k)\right)+\epsilon k \frac{\left[x_{\mathrm{m}}(k)-\langle x\rangle\right]^{2}}{2}\right\},
\end{aligned}
$$

which depends on the degree $k$ of the element and on the mean field $\langle x\rangle$.

Figure 8 depicts the typical dependence of the potential barrier $V_{\mathrm{u}}(\langle x\rangle ; k)$ on the degree $k$ for $\langle x\rangle=0(\mathrm{a}),\langle x\rangle=0.3$ (b), $\langle x\rangle=0.6$ (c), and $\langle x\rangle=0.9$ (d), and for various values of the coupling intensity $\epsilon$. It is observed that $V_{\mathrm{u}}(\langle x\rangle ; k)$ decreases approximately linearly with the degree $k$, so the escape time decreases exponentially with $k$. Such linear dependence of $V_{\mathrm{u}}(\langle x\rangle ; k)$ on $k$ is generally observed in the range $-1<\langle x\rangle<$ 1 , and the largest value of the potential barrier $V_{\mathrm{u}}\left(\langle x\rangle ; k_{\min }\right)$ tends to decrease as the amplitude of the mean field $|\langle x\rangle|$ increases. Therefore, as the system becomes more ordered and $|\langle x\rangle|$ increases, the elements escape from the metastable state is accelerated.

The escape rate argument provides a qualitative explanation for the consecutive relaxation process in the order of degrees, as well as the large differences observed between relaxation times of the elements with different degrees.

\section{Total relaxation time of the system}

Figure 9 shows the evolution of the overall mean field $\bar{x}(t)$ of the network and $\langle x\rangle(t)$ of the NLFPE for various values of coupling intensity $\epsilon$. As can be observed in Fig. 9(a), the relaxation is much faster when $\epsilon$ is larger, due to the fact that the potential barrier $V_{\mathrm{u}}(\langle x\rangle ; k)$ is reduced for larger $\epsilon$. Figure 9(b) shows the total relaxation times for the original network system and of the NLFPE with respect to $\epsilon$ and compares them with (a)

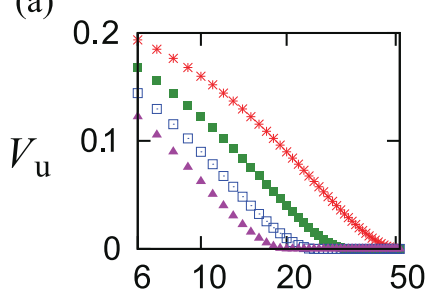

(c)

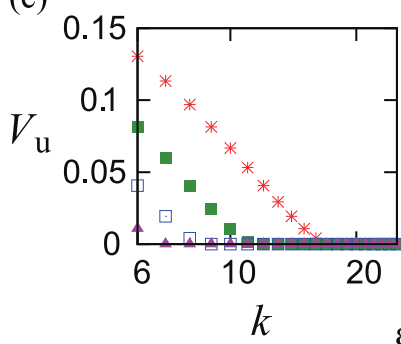

(b)

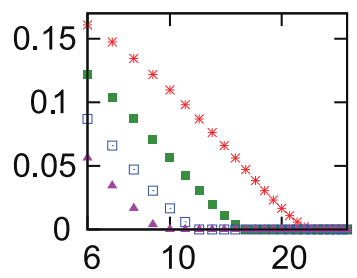

(d)

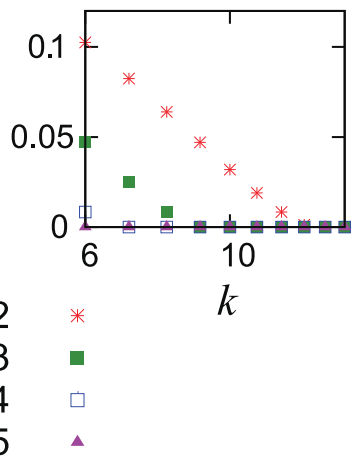

FIG. 8. (Color online) Dependence of the potential barrier $V_{\mathrm{u}}$ on the degree $k$ for $\langle x\rangle=0$ (a), $\langle x\rangle=0.3$ (b), $\langle x\rangle=0.6$ (c), and $\langle x\rangle=0.9(\mathrm{~d})$.

results obtained using the Kramers' theory. From the escape rate argument, we can expect the most time-consuming process to be the relaxation of the elements with the lowest degree $k=$ $k_{\text {min }}$ and therefore this dominates the total relaxation time of the whole system. We consider a situation where all elements other than those with the lowest degree $k_{\min }$ have already relaxed to the upper group near $x=x^{*} \approx 1$. The value of the mean field can then be approximately calculated as

$$
\bar{x} \simeq \sum_{k=k_{\min }+1}^{k_{\max }} \frac{N(k)}{N} \frac{k}{\langle k\rangle} x^{*} \approx 0.8841
$$

for the present situation with $\gamma=2.5$ and $k_{\max }=87$. The dashed line in Fig. 9(b) indicates the Kramers' escape time, $T=C \exp \left[-V_{\mathrm{u}}\left(\bar{x} ; k_{\min }\right) / D\right]$ for the elements with $k=k_{\min }$, where the prefactor $C$ is determined by the least-squares fit. The total relaxation times for the network and of the NLFPE are fitted well by the longest escape time $T$ of the elements with the lowest degree, $k_{\min }$. This result also supports the theory that the escape rate argument can qualitatively explain the relaxation dynamics of the system.

\section{CONCLUSIONS AND DISCUSSION}

In this paper, we studied the phase ordering process of diffusively coupled noisy bistable elements on scale-free networks. We demonstrated that an order-disorder transition similar to that of globally coupled systems also occurs in a network-organized system, and we revealed the characteristic degree dependence of the behavior. The mean-field approximation of the network was used to show that network patterns can be fitted using the stable fixed points of the local dynamics of individual elements that are subjected to the mean field 


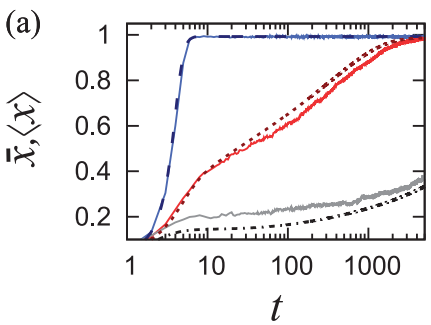

network $\varepsilon=0.2$ network $\varepsilon=0.02$ network $\varepsilon=0.005$ NLFPE $\varepsilon=0.2 \quad-\quad$ NLFPE $\varepsilon=0.02 \quad \ldots \ldots .$.

(b)

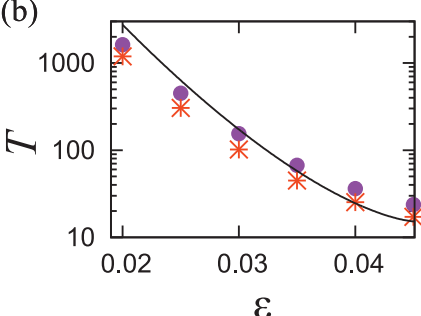

\section{ACKNOWLEDGMENTS}

We gratefully acknowledge A. S. Mikhailov for useful discussions and insightful comments. Y.A. is supported by the JSPS Research Fellowships for Young Scientists. S.H. acknowledges JSPS Fellowship for Research Abroad. H.N. thanks KAKENHI $(22684020,25540108)$ from JSPS, CREST Kokubu project from JST, and the FIRST Aihara project from JSPS for financial support.

\section{APPENDIX A: EXISTENCE OF SELF-CONSISTENT SOLUTIONS}

We here show that

$$
\left.\frac{\partial x^{(1)}}{\partial x^{(0)}}\right|_{x^{(0)}=0}<1 \text { when } D \rightarrow \infty
$$

FIG. 9. (Color online) (a) Evolution of the mean field $\bar{x}(t)$ during the relaxation of the original network system $(N=1800$, averaged over 50 realizations) and the mean field $\langle x\rangle(t)$ of the approximate NLFPE at several values of $\epsilon$. (b) Relaxation time $T$ of the original network system ( $N=1800$, averaged over 50 realizations) and of the NLFPE, which is defined as the time taken for the overall mean field $\bar{x}(t)$ or $\langle x\rangle(t)$ to exceed $0.95 \times x^{*}$, plotted with respect to $\epsilon$. The dashed line is the Kramers' escape time $T=C \exp \left[-V_{\mathrm{u}}\left(\bar{x} ; k_{\min }\right) / D\right]$ with $\bar{x}$ of Eq. (19), where the prefactor $C$ is determined by the leastsquares fit. The network parameters are $k_{\min }=6, k_{\max }=87$, and $\gamma=2.5$, and the noise intensity is fixed at $D=0.02$.

with degree-dependent coupling intensity in a late stage of evolution. Then, using the NLFPE approximation of the original system and the self-consistency analysis, we argued that an order-disorder transition occurs in the original network system and that the noise intensity acts as a bifurcation parameter in the system. We also found that the relaxation of the system toward the stationary state takes place consecutively in order of the degrees and qualitatively explained the dependence on the degrees based on the Kramers' escape rate theory.

Though we are not considering specific real-world phenomena in the present study, we note that bistability often arise in models of social consensus formation. For example, the Sznajd model [48], a well-known model of opinion formation in a community that can reproduce empirical scaling properties of real elections [49], behaves like a single bistable system under certain conditions [50]. Thus, if such communities are connected to form a network structure (see, e.g., Ref. [37] for the majority model on two coupled networks), their dynamics would be described by the network-organized bistable systems similar to that we analyzed in the present study.

The strong dependence of the network dynamics on the degree of the elements is commonly observed in various models with scale-free network structures, which is also the case in the present study. Though the focus of the present study is on generic dynamical properties of the class of coupled bistable dynamical systems on scale-free networks and not on specific real-world systems, our theoretical findings may be of potential relevance to the analysis of real-world network dynamics.

$$
\left.\frac{\partial x^{(1)}}{\partial x^{(0)}}\right|_{x^{(0)}=0}>1 \text { when } D \rightarrow+0,
$$

by generalizing the argument developed by Shiino [7] for globally coupled systems, where $x^{(0)}$ and $x^{(1)}$ represent the presumed and resulting values of the mean field, respectively. The stationary PDF $P_{\text {st }}(x ; k)$ in Eq. (13) with $\langle x\rangle=x^{(0)}$, which satisfies the normalization condition $\int P_{\mathrm{st}}(x ; k) d x=1$, can explicitly be written as

$$
\begin{aligned}
P_{\mathrm{st}}(x ; k) & =\frac{1}{z_{P}(k)} \exp \left[-\frac{V_{0}(x)}{D}-\epsilon k \frac{\left(x-x^{(0)}\right)^{2}}{2 D}\right], \\
z_{P}(k) & =\int \exp \left[-\frac{V_{0}(x)}{D}-\epsilon k \frac{\left(x-x^{(0)}\right)^{2}}{2 D}\right] d x,
\end{aligned}
$$

where $z_{P}(k)$ is the inverse of the normalization constant. The resulting mean field $x^{(1)}$ defined in Eq. (14) can be expressed as

$$
\begin{aligned}
& x^{(1)}=\sum_{k} \frac{k}{\langle k\rangle} r(k) x_{k}^{(1)}, \\
& x_{k}^{(1)}=\langle x\rangle_{k}=\int x P_{\mathrm{st}}(x ; k) d x,
\end{aligned}
$$

where $\langle\cdot\rangle_{k}$ denotes the expectation with respect to $P_{\mathrm{st}}(x ; k)$. We first show that

$$
\left.\frac{\partial x^{(1)}}{\partial x^{(0)}}\right|_{x^{(0)}=0}=\left.\sum_{k=k_{\min }}^{k_{\max }} \frac{k}{\langle k\rangle} r(k) \frac{\epsilon k}{D}\left\langle x^{2}\right\rangle_{k}\right|_{x^{(0)}=0},
$$

where $\left\langle x^{2}\right\rangle_{k}=\int x^{2} P_{\mathrm{st}}(x ; k) d x$. Differentiating the definition of $x^{(1)}$ by $x^{(0)}$ yields

$$
\frac{\partial x^{(1)}}{\partial x^{(0)}}=\sum_{k=k_{\min }}^{k_{\max }} \frac{k}{\langle k\rangle} r(k) \frac{\partial x_{k}^{(1)}}{\partial x^{(0)}},
$$

where

$$
\begin{aligned}
\frac{\partial x_{k}^{(1)}}{\partial x^{(0)}}= & \frac{1}{z_{P}(k)^{2}} \int\left\{z_{P}(k) \frac{\partial}{\partial x^{(0)}}-\left(\frac{\partial z_{P}(k)}{\partial x^{(0)}}\right)\right\} \\
& \times x \exp \left[-\frac{V_{0}(x)}{D}-\epsilon k \frac{\left(x-x^{(0)}\right)^{2}}{2 D}\right] d x,
\end{aligned}
$$


and the derivative of $z_{P}(k)$ can be calculated as

$$
\begin{aligned}
\frac{\partial z_{P}(k)}{\partial x^{(0)}} & =\frac{\epsilon k}{D} \int\left(x-x^{(0)}\right) \exp \left[-\frac{V_{0}(x)}{D}-\epsilon k \frac{\left(x-x^{(0)}\right)^{2}}{2 D}\right] d x \\
& =\frac{\epsilon k}{D} z_{P}(k) \int\left(x-x^{(0)}\right) P_{\mathrm{st}}(x ; k) d x \\
& =\frac{\epsilon k}{D} z_{P}(k)\left(x_{k}^{(1)}-x^{(0)}\right) .
\end{aligned}
$$

Thus, we obtain

$$
\begin{aligned}
\frac{\partial x_{k}^{(1)}}{\partial x^{(0)}}= & \frac{\epsilon k}{D} \frac{1}{z_{P}(k)} \int\left(x^{2}-x x_{k}^{(1)}\right) \\
& \times \exp \left[-\frac{V_{0}(x)}{D}-\epsilon k \frac{\left(x-x^{(0)}\right)^{2}}{2 D}\right] d x \\
= & \frac{\epsilon k}{D} \int\left(x^{2}-x x_{k}^{(1)}\right) P_{\mathrm{st}}(x ; k) d x \\
= & \frac{\epsilon k}{D}\left\{\left\langle x^{2}\right\rangle_{k}-\left(x_{k}^{(1)}\right)^{2}\right\} \\
= & \frac{\epsilon k}{D}\left(\left\langle x^{2}\right\rangle_{k}-\langle x\rangle_{k}^{2}\right) .
\end{aligned}
$$

From this result and the fact that $\langle x\rangle_{k}^{2}=0$ when $x^{(0)}=0$, Eq. (A5) follows.

We now show that

$$
\begin{aligned}
\left.\frac{\partial x^{(1)}}{\partial x^{(0)}}\right|_{x^{(0)}=0} & =\left.\sum_{k=k_{\min }}^{k_{\max }} \frac{k}{\langle k\rangle} r(k) \frac{\epsilon k}{D}\left\langle x^{2}\right\rangle_{k}\right|_{x^{(0)}=0}<1 \\
\text { when } \quad D & \rightarrow \infty
\end{aligned}
$$

and

$$
\begin{aligned}
\left.\frac{\partial x^{(1)}}{\partial x^{(0)}}\right|_{x^{(0)}=0} & =\left.\sum_{k=k_{\min }}^{k_{\max }} \frac{k}{\langle k\rangle} r(k) \frac{\epsilon k}{D}\left\langle x^{2}\right\rangle_{k}\right|_{x^{(0)}=0}>1 \\
\text { when } \quad D & \rightarrow+0,
\end{aligned}
$$

hold, which we used in the main text.

We first consider Eq. (A10). In order to confirm this inequality, we show that $\left.(\epsilon k / D)\left\langle x^{2}\right\rangle_{k}\right|_{x^{(0)}=0} \rightarrow 0$ as $D \rightarrow \infty$ for finite $k$. By definition, $\left.(1 / D)\left\langle x^{2}\right\rangle_{k}\right|_{x^{(0)}=0}$ is expressed as

$$
\begin{aligned}
\left.\frac{1}{D}\left\langle x^{2}\right\rangle_{k}\right|_{x^{(0)}=0} & =\frac{1}{D} \frac{\int x^{2} \exp \left[-\frac{V_{0}(x)}{D}-\epsilon k \frac{x^{2}}{2 D}\right] d x}{\int \exp \left[-\frac{V_{0}(x)}{D}-\epsilon k \frac{x^{2}}{2 D}\right] d x} \\
& =\frac{1}{D} \frac{\int x^{2} \exp (\Phi / D) d x}{\int \exp (\Phi / D) d x}
\end{aligned}
$$

where

$$
\Phi(x) \equiv-V_{0}(x)-\epsilon k \frac{x^{2}}{2}
$$

is defined. Since $V_{0}(x)$ is a quartic function in $x$, we can introduce three constants, $M, K_{1}$, and $K_{2}$, such that

$$
-K_{2} x^{4}-M \leqslant \Phi(x) \leqslant-K_{1} x^{4}+M
$$

is satisfied. Using Eq. (A14), we can show that

$$
\begin{aligned}
\frac{\int x^{2} \exp (\Phi / D) d x}{\int \exp (\Phi / D) d x} & \leqslant \frac{\int x^{2} \exp \left[\frac{-K_{1} x^{4}+M}{D}\right] d x}{\int \exp \left[\frac{-K_{2} x^{4}-M}{D}\right] d x} \\
& =\frac{\exp (2 M / D) D^{1 / 2} \int X^{2} \exp \left(-K_{1} X^{4}\right) d X}{\int \exp \left(-K_{2} X^{4}\right) d X},
\end{aligned}
$$

where we defined $X=D^{-1 / 4} x$. Thus,

$$
\begin{aligned}
0 & \leqslant\left.\lim _{D \rightarrow \infty} \frac{1}{D}\left\langle x^{2}\right\rangle_{k}\right|_{x^{(0)}=0} \\
& \leqslant \lim _{D \rightarrow \infty} \exp (2 M / D) D^{-1 / 2} \frac{\int X^{2} \exp \left(-K_{1} X^{4}\right) d X}{\int \exp \left(-K_{2} X^{4}\right) d X}=0
\end{aligned}
$$

holds, i.e.,

$$
\left.\lim _{D \rightarrow \infty} \frac{\epsilon k}{D}\left\langle x^{2}\right\rangle_{k}\right|_{x^{(0)}=0}=0
$$

for finite $k$. Therefore, for finite $k_{\max }$,

$$
\left.\lim _{D \rightarrow \infty} \sum_{k=k_{\min }}^{k_{\max }} \frac{k}{\langle k\rangle} r(k) \frac{\epsilon k}{D}\left\langle x^{2}\right\rangle_{k}\right|_{x^{(0)}=0}=0
$$

holds, so Eq. (A10) is satisfied.

Next, we show that Eq. (A11) asymptotically holds in the limit $D \rightarrow+0$ for finite $k$. We first consider the case that $\Phi(x)$ has two extrema at $x \neq 0(\epsilon k<1)$ and denote the location of the extrema as $x=\left(x_{\mathrm{S}}\right)_{i}(i=1,2)$. We expand $\Phi(x)$ in a Taylor series around $\left(x_{\mathrm{S}}\right)_{i}$ as

$$
\Phi(x)=A-a_{i}\left[x-\left(x_{S}\right)_{i}\right]^{2}+\cdots,
$$

where

$$
a_{i}>0, \quad A=\max _{x} \Phi(x) .
$$

Using the saddle approximation

$$
\begin{aligned}
\int f(x) e^{K \Phi(x)} d x= & \sum_{i} e^{K \Phi\left[\left(x_{\mathrm{S}}\right)_{i}\right]} \times \sqrt{\frac{2 \pi}{K\left|\Phi^{\prime \prime}\left[\left(x_{\mathrm{S}}\right)_{i}\right]\right|}} \\
& \times\left\{f\left[\left(x_{\mathrm{S}}\right)_{i}\right]+O\left(K^{-1}\right)\right\}
\end{aligned}
$$$$
\text { when } K \rightarrow \infty
$$

with $f(x)=x^{2}$ or $f(x)=1, K=1 / D,\left|\Phi^{\prime \prime}\left[\left(x_{\mathrm{S}}\right)_{i}\right]\right|=2 a_{i}$, and $\Phi\left[\left(x_{\mathrm{S}}\right)_{i}\right]=A$, we can show that

$$
\begin{aligned}
\left.\left\langle x^{2}\right\rangle_{k}\right|_{x^{(0)}=0} & \rightarrow \frac{e^{A / D} \sum_{i=1}^{2}\left(\frac{D \pi}{a_{i}}\right)^{1 / 2}\left\{\left(x_{\mathrm{S}}\right)_{i}^{2}+O(D)\right\}}{e^{A / D} \sum_{i=1}^{2}\left(\frac{D \pi}{a_{i}}\right)^{1 / 2}\{1+O(D)\}} \\
& =\frac{\sum_{i=1}^{2}\left(\frac{1}{a_{i}}\right)^{1 / 2}\left\{\left(x_{\mathrm{S}}\right)_{i}^{2}+O(D)\right\}}{\sum_{i=1}^{2}\left(\frac{1}{a_{i}}\right)^{1 / 2}\{1+O(D)\}} \\
& >0
\end{aligned}
$$

is asymptotically satisfied in the limit $D \rightarrow+0$. Therefore, for finite $k$,

$$
\left.\lim _{D \rightarrow+0} \frac{\epsilon k}{D}\left\langle x^{2}\right\rangle_{k}\right|_{x^{(0)}=0}=\infty
$$

holds. We next consider the case that $\Phi(x)$ takes a single maximum at $x=0$, i.e., when $\epsilon k>1$, where $k$ is assumed to be finite. In this case, by defining $X=(\epsilon k / D)^{1 / 2} x$, $\left.(\epsilon k / D)\left\langle x^{2}\right\rangle_{k}\right|_{x^{(0)}=0}$ can be transformed as

$$
\begin{aligned}
& \left.\lim _{D \rightarrow+0} \frac{\epsilon k}{D}\left\langle x^{2}\right\rangle_{k}\right|_{x^{(0)}=0} \\
& =\lim _{D \rightarrow+0} \frac{\epsilon k}{D} \frac{\int x^{2} \exp \left[\left(\frac{1}{2 D}-\frac{\epsilon k}{2 D}\right) x^{2}-\frac{1}{4 D} x^{4}\right] d x}{\int \exp \left[\left(\frac{1}{2 D}-\frac{\epsilon k}{2 D}\right) x^{2}-\frac{1}{4 D} x^{4}\right] d x}
\end{aligned}
$$




$$
\begin{aligned}
& =\lim _{D \rightarrow+0} \frac{\int X^{2} \exp \left[-\frac{1}{2}\left(1-\frac{1}{\epsilon k}\right) X^{2}-\frac{D X^{4}}{4 \epsilon^{2} k^{2}}\right] d X}{\int \exp \left[-\frac{1}{2}\left(1-\frac{1}{\epsilon k}\right) X^{2}-\frac{D X^{4}}{4 \epsilon^{2} k^{2}}\right] d X} \\
& =\frac{\int X^{2} \exp \left[-\frac{1}{2}\left(1-\frac{1}{\epsilon k}\right) X^{2}\right] d X}{\int \exp \left[-\frac{1}{2}\left(1-\frac{1}{\epsilon k}\right) X^{2}\right] d X} .
\end{aligned}
$$

Performing the Gaussian integral, we can show that

$$
\left.\lim _{D \rightarrow+0} \frac{\epsilon k}{D}\left\langle x^{2}\right\rangle_{k}\right|_{x^{(0)}=0}=\sqrt{\frac{1}{1-\frac{1}{\epsilon k}}}=\sqrt{\frac{\epsilon k}{\epsilon k-1}}>1 .
$$

From this result and Eq. (A23), we can show that $(\epsilon k / D)$ $\left.\left\langle x^{2}\right\rangle_{k}\right|_{x^{(0)}=0}>1$ for arbitrary $k$, i.e., Eq. (A11) is satisfied.

\section{APPENDIX B: LYAPUNOV FUNCTIONAL}

Following Shiino and Frank et al. [7,32], we define a Lyapunov functional $I$ as

$$
I=\frac{H}{D}-S,
$$

where $S$ and $H$ are expectations of the information entropy and the energy, respectively, and $D$ is the noise intensity. If we define $F=D I=H-D S, F$ can be considered a generalized free energy. For the NLFPE approximating the original network model, $S$ and $H$ are given as follows:

$$
\begin{aligned}
S & =-\sum_{\ell} r(\ell) \int P(x, t ; \ell) \ln P(x, t ; \ell) d x, \\
H & =\sum_{\ell} r(\ell) \int P(x, t ; \ell)\left\{V_{0}(x)+\frac{\epsilon \ell}{2\langle k\rangle} \sum_{m} m r(m) \int P(y, t ; m) \frac{(x-y)^{2}}{2} d y\right\} d x,
\end{aligned}
$$

where $V_{0}(x)$ is the potential defined in Eq. (13). This gives

$$
I=\sum_{\ell} r(\ell) \int P(x, t ; \ell)\left[\ln P(x, t ; \ell)+\frac{V_{0}(x)}{D}\right] d x+\frac{\epsilon}{2 D\langle k\rangle} \sum_{\ell} \ell r(\ell) \sum_{m} m r(m) \iint P(x, t ; \ell) P(y, t ; m) \frac{(x-y)^{2}}{2} d x d y,
$$

which we presented as Eq. (15) in the main text. Below we show that this $I$ is a Lyapunov functional of the system, i.e., $I$ decreases monotonically as the system evolves and reaches a minimum value when the system becomes stationary, following the treatment by Frank et al. [32].

To calculate the derivative of $I$, we additionally define the following two functions. The first function $Q(x ; k)$ is the probability density function (PDF) of the NLFPE, i.e., $P_{\mathrm{st}}(x ; k)$ of Eq. (13) with a self-consistent mean field $x^{*}$. The second function is the inverse of the normalization constant of $Q(x ; k)$, which can be expressed as

$$
\tilde{z}_{Q}(k)=\frac{\tilde{Q}(x ; k)}{Q(x ; k)},
$$

i.e., $Q(x ; k)=\tilde{Q}(x ; k) / \tilde{z}_{Q}(k)$, where $\tilde{Q}(x ; k)$ is a stationary PDF of the NLFPE without normalization,

$$
\begin{aligned}
\tilde{Q}(x ; k) & =\exp \left[-\frac{V_{0}(x)}{D}-\frac{\epsilon k}{D\langle k\rangle} \sum_{m} m r(m) \int Q(y ; m) \frac{(x-y)^{2}}{2} d y\right] \\
& =\exp \left[-\frac{V_{0}(x)}{D}-\epsilon k \frac{\left(x-x^{*}\right)^{2}}{2 D}-\frac{\epsilon k}{D\langle k\rangle} \sum_{m} m r(m) \int Q(y ; m) \frac{\left(x^{*}-y\right)^{2}}{2} d y\right] .
\end{aligned}
$$

Since the last term in the second line is constant, we can see that $\tilde{Q}(x ; k)$ is proportional to Eq. (13) with $\langle x\rangle=x^{*}$. Thus, the inverse of the normalization constant can be expressed as

$$
\tilde{z}_{Q}(k)=\frac{\exp \left[-V_{0}(x) / D\right]}{f[Q(x ; k) ; k]},
$$

where $f[Q(x ; k) ; k]$ is defined as

$$
f[Q(x ; k) ; k]=Q(x ; k) \exp \left[\frac{\epsilon k}{D\langle k\rangle} \sum_{m} m r(m) \int Q(y ; m) \frac{(x-y)^{2}}{2} d y\right] .
$$

This function projects $Q(x ; k)$ onto a stationary PDF ( $\left.\propto \exp \left[-V_{0}(x) / D\right]\right)$ of the uncoupled Fokker-Planck equation, i.e., Eq. (12) with $\epsilon=0$ [32]. Thus, the potential $V_{0}(x)$ can be expressed as

$$
V_{0}(x)=-D \ln \left\{\tilde{z}_{Q}(k) f[Q(x ; k) ; k]\right\} .
$$


Using the functions $\tilde{z}_{Q}(k)$ and $f[Q(x ; k) ; k]$ defined above, $H$ can be written as

$$
\begin{aligned}
H= & -D \sum_{\ell} r(\ell) \int P(x, t ; \ell) \ln \left\{\tilde{z}_{Q}(\ell) f[Q(x ; \ell) ; \ell]\right\} d x+\frac{\epsilon}{2\langle k\rangle} \sum_{\ell} \ell r(\ell) \sum_{m} m r(m) \iint P(x, t ; \ell) P(y, t ; m) \frac{(x-y)^{2}}{2} d x d y \\
= & -D \sum_{\ell} r(\ell) \int P(x, t ; \ell) \ln f[Q(x ; \ell) ; \ell] d x-D \sum_{\ell} r(\ell) \ln \tilde{z}_{Q}(\ell) \\
& +\frac{\epsilon}{2\langle k\rangle} \sum_{\ell} \ell r(\ell) \sum_{m} m r(m) \iint P(x, t ; \ell) P(y, t ; m) \frac{(x-y)^{2}}{2} d x d y
\end{aligned}
$$

where we used $\int P(x, t ; \ell) d x=1$. Thus, the functional $I$ can be written as

$$
\begin{aligned}
I[P(x, t ; k), Q(x ; k)]= & \frac{\epsilon}{2 D\langle k\rangle} \sum_{\ell} \ell r(\ell) \sum_{m} m r(m) \iint P(x, t ; \ell) P(y, t ; m) \frac{(x-y)^{2}}{2} d x d y+\sum_{\ell} r(\ell) \int P(x, t ; \ell) \ln P(x, t ; \ell) d x \\
& -\sum_{\ell} r(\ell) \int P(x, t ; \ell) \ln f[Q(x ; \ell) ; \ell] d x-\sum_{\ell} r(\ell) \ln \tilde{z}_{Q}(\ell) .
\end{aligned}
$$

Here, the last term is a finite constant independent of $x$, so it can be removed without violating the conditions of the Lyapunov functional.

We now prove that $I[P(x, t ; k), Q(x ; k)]$ is a Lyapunov functional, i.e., I satisfies the following conditions:

$$
\frac{d}{d t} I \leqslant 0, \quad \frac{d}{d t}=0 \Leftrightarrow P(x, t ; k)=P_{\mathrm{st}}(x ; k), \quad I>-\infty
$$

First, let us confirm that $I$ decreases monotonously. Differentiating $I$ by the time $t$ yields

$$
\begin{aligned}
\frac{d}{d t} I(t)= & \frac{\epsilon}{D\langle k\rangle} \sum_{\ell} \ell r(\ell) \sum_{m} m r(m) \iint \frac{\partial P(x, t ; \ell)}{\partial t} P(y, t ; m) \frac{(x-y)^{2}}{2} d x d y \\
& +\sum_{\ell} r(\ell) \int \frac{\partial P(x, t ; \ell)}{\partial t} \ln P(x, t ; \ell) d x-\sum_{\ell} r(\ell) \int \frac{\partial P(x, t ; \ell)}{\partial t} \ln f[Q(x ; \ell) ; \ell] d x \\
= & \sum_{\ell} r(\ell) \int \frac{\partial P(x, t ; \ell)}{\partial t}\left\{\frac{\epsilon \ell}{D\langle k\rangle} \sum_{m} m r(m) \int P(y, t ; m) \frac{(x-y)^{2}}{2} d y+\ln P(x, t ; \ell)-\ln f[Q(x ; \ell) ; \ell]\right\} d x,
\end{aligned}
$$

where we used $\int\{\partial P(x, t ; k) / \partial t\} d x=(d / d t) \int P(x, t ; k) d x=0$. By plugging $P(x, t ; k)$ as $Q(x ; k)$ into Eq. (B7), we obtain

$$
\ln P(x, t ; k)+\frac{\epsilon k}{D\langle k\rangle} \sum_{m} m r(m) \int P(y, t ; m) \frac{(x-y)^{2}}{2} d y=\ln f[P(x, t ; k) ; k] .
$$

Thus, Eq. (B12) can be simplified as

$$
\frac{d}{d t} I(t)=\sum_{\ell} r(\ell) \int \frac{\partial P(x, t ; \ell)}{\partial t} \ln \left\{\frac{f[P(x, t ; \ell) ; \ell]}{f[Q(x ; \ell) ; \ell]}\right\} d x .
$$

Here, $\partial P / \partial t$ can be expressed using the NLFPE Eq. (12) as

$$
\begin{aligned}
\frac{\partial P(x, t ; k)}{\partial t} & =-\frac{\partial}{\partial x} P(x, t ; k)\left\{h(x)+\epsilon k\left[\sum_{m} \frac{m}{\langle k\rangle} r(m) \int y P(y, t ; m) d y\right\}-x\right]+D \frac{\partial^{2}}{\partial x^{2}} P(x, t ; k) \\
& =-\frac{\partial}{\partial x} P(x, t ; k)\left\{h(x)-\frac{\epsilon k}{\langle k\rangle} \sum_{m} m r(m) \int(x-y) P(y, t ; m) d y-D \frac{\partial}{\partial x} \ln P(x, t ; k)\right\} \\
& =-\frac{\partial}{\partial x} P(x, t ; k)\left\{h(x)-D \frac{\partial}{\partial x} \ln f[P(x, t ; k) ; k]\right\},
\end{aligned}
$$

where $h(x)=x-x^{3}$ can be expressed as

$$
h(x)=-\frac{\partial V_{0}(x)}{\partial x}=D \frac{\partial}{\partial x}\left\{\ln \tilde{z}_{Q}(k)+\ln f[Q(x ; k) ; k]\right\}=D \frac{\partial}{\partial x} \ln f[Q(x ; k) ; k] .
$$

Using Eq. (B16), Eq. (B15) can be expressed as follows:

$$
\frac{\partial P(x, t ; k)}{\partial t}=D \frac{\partial}{\partial x} P(x, t ; k) \frac{\partial}{\partial x} \ln \left\{\frac{f[P(x, t ; k) ; k]}{f[Q(x ; k) ; k]}\right\} .
$$


Therefore, we can show that the Lyapunov functional $I$ decreases monotonously with time, i.e.,

$$
\begin{aligned}
\frac{d}{d t} I(t)= & D \sum_{\ell} r(\ell) \int\left[\frac{\partial}{\partial x} P(x, t ; \ell) \frac{\partial}{\partial x} \ln \left\{\frac{f[P(x, t ; \ell) ; \ell]}{f[Q(x ; \ell) ; \ell]}\right\}\right] \ln \left\{\frac{f[P(x, t ; \ell) ; \ell]}{f[Q(x ; \ell) ; \ell]}\right\} d x \\
= & D \sum_{\ell} r(\ell)\left[P(x, t ; \ell)\left(\frac{\partial}{\partial x} \ln \left\{\frac{f[P(x, t ; \ell) ; \ell]}{f[Q(x ; \ell) ; \ell]}\right\}\right) \ln \left\{\frac{f[P(x, t ; \ell) ; \ell]}{f[Q(x ; \ell) ; \ell]}\right\}\right]_{-\infty}^{\infty} \\
& -D \sum_{\ell} r(\ell) \int P(x, t ; \ell)\left(\frac{\partial}{\partial x} \ln \left\{\frac{f[P(x, t ; \ell) ; \ell]}{f[Q(x ; \ell) ; \ell]}\right\}\right) \frac{\partial}{\partial x} \ln \left\{\frac{f[P(x, t ; \ell) ; \ell]}{f[Q(x ; \ell) ; \ell]}\right\} d x \\
= & -D \sum_{\ell} r(\ell) \int P(x, t ; \ell)\left[\frac{\partial}{\partial x} \ln \left\{\frac{f[P(x, t ; \ell) ; \ell]}{f[Q(x ; \ell) ; \ell]}\right\}\right]^{2} d x \leqslant 0 .
\end{aligned}
$$

Next, we show that $\frac{d I}{d t}=0 \Leftrightarrow P(x, t ; k)=P_{\mathrm{st}}(x ; k)$. Since it is clear that $P(x, t ; k)=P_{\mathrm{st}}(x ; k) \Rightarrow \frac{d I}{d t}=0$, we only show its converse. From Eq. (B18),

$$
\frac{\partial}{\partial x} \ln \left\{\frac{f[P(x, t ; k) ; k]}{f[Q(x ; k) ; k]}\right\}=0
$$

should hold for arbitrary $k$ and $x$ when $\frac{d I}{d t}=0$. Namely $f[P(x, t ; k) ; k] / f[Q(x ; k) ; k]$, which can be expressed as

$$
\frac{f[P(x, t ; k) ; k]}{f[Q(x ; k) ; k]}=\tilde{z}_{Q}(k) P(x, t ; k) \exp \left[\frac{V_{0}(x)}{D}+\frac{\epsilon k}{D\langle k\rangle} \sum_{m} m r(m) \int P(y, t ; m) \frac{(x-y)^{2}}{2} d y\right],
$$

should be constant. Thus, $P(x, t ; k)$ should satisfy

$$
P(x, t ; k) \propto \exp \left[-\frac{V_{0}(x)}{D}-\frac{\epsilon k}{D\langle k\rangle} \sum_{m} m r(m) \int P(y, t ; m) \frac{(x-y)^{2}}{2} d y\right] \propto \exp \left[-\frac{V_{0}(x)}{D}-\epsilon k \frac{(x-\langle x\rangle)^{2}}{2 D}\right],
$$

which gives the stationary PDF $P_{\mathrm{st}}(x ; k)$ of Eq. (13). As we argued in Sec. IV A, $P_{\mathrm{st}}(x ; k)$ should satisfy $\langle x\rangle=x^{*}$. When the system is in the ordered phase, two values of $x^{*}$ are possible and the NLFPE can possess two different stationary PDFs, $P_{\mathrm{st}}^{(1)}(x ; k)=Q(x ; k)$ and $P_{\mathrm{st}}^{(2)}(x ; k)$. A stationary PDF $P_{\mathrm{st}}(x ; k)$ corresponds to either of these stationary PDFs and may not agree with $Q(x ; k)$. See Frank et al. [32] for details.

Finally, we show that $I>-\infty$. Using $\langle x\rangle=(1 /\langle k\rangle) \sum_{m} m r(m) \int y P(y, t ; m) d y$ and $(x-y)^{2}=(x-\langle x\rangle)^{2}+2(x-$ $\langle x\rangle)(\langle x\rangle-y)+(\langle x\rangle-y)^{2}$, it can be shown that

$$
\begin{aligned}
\frac{1}{\langle k\rangle} \sum_{m} m r(m) \int P(y, t ; m) \frac{(x-y)^{2}}{2} d y= & \frac{(x-\langle x\rangle)^{2}}{2}+(x-\langle x\rangle)\left(\langle x\rangle-\frac{1}{\langle k\rangle} \sum_{m} m r(m) \int y P(y, t ; m) d y\right) \\
& +\frac{1}{\langle k\rangle} \sum_{m} m r(m) \int P(y, t ; m) \frac{(\langle x\rangle-y)^{2}}{2} d y \\
= & \frac{(x-\langle x\rangle)^{2}}{2}+\frac{1}{\langle k\rangle} \sum_{m} m r(m) \int \frac{(\langle x\rangle-y)^{2}}{2} P(y, t ; m) d y .
\end{aligned}
$$

Thus, the Lyapunov functional of Eq. (15) can be rewritten as

$$
\begin{aligned}
I[P(x, t ; k), Q(x ; k)]= & \sum_{\ell} \ell r(\ell) \int P(x, t ; \ell)\left\{\epsilon \frac{(x-\langle x\rangle)^{2}}{4 D}+\frac{\epsilon}{2 D\langle k\rangle} \sum_{m} m r(m) \int P(y, t ; m) \frac{(\langle x\rangle-y)^{2}}{2} d y\right\} d x \\
& +\sum_{\ell} r(\ell) \int P(x, t ; \ell) \ln P(x, t ; \ell) d x+\sum_{\ell} r(\ell) \int P(x, t ; \ell) \frac{V_{0}(x)}{D} d x \\
= & \sum_{\ell} \ell r(\ell) \int P(x, t ; \ell) \epsilon \frac{(x-\langle x\rangle)^{2}}{4 D} d x+\sum_{m} m r(m) \int P(y, t ; m) \epsilon \frac{(\langle x\rangle-y)^{2}}{4 D} d y \\
& +\sum_{\ell} r(\ell) \int P(x, t ; \ell) \ln P(x, t ; \ell) d x+\sum_{\ell} r(\ell) \int P(x, t ; \ell) \frac{V_{0}(x)}{D} d x \\
= & \sum_{\ell} r(\ell) \int P(x, t ; \ell)\left\{\frac{V_{0}(x)}{D}+\epsilon \ell \frac{(x-\langle x\rangle)^{2}}{2 D}\right\} d x+\sum_{\ell} r(\ell) \int P(x, t ; \ell) \ln P(x, t ; \ell) d x .
\end{aligned}
$$


By introducing

$$
P^{\prime}(x ; \ell)=\frac{1}{z_{P^{\prime}}(\ell)} \exp \left[-\frac{V_{0}(x)}{D}-\epsilon \ell \frac{(x-\langle x\rangle)^{2}}{2 D}\right]
$$

with a normalization constant $z_{P^{\prime}}(\ell)^{-1}$, which does not necessarily satisfy the self-consistency condition, $I$ can further be rewritten as

$$
\begin{aligned}
I[P(x, t ; k), Q(x ; k)] & =-\sum_{\ell} r(\ell) \int P(x, t ; \ell)\left\{\ln P^{\prime}(x ; \ell)+\ln z_{P}(\ell)\right\} d x+\sum_{\ell} r(\ell) \int P(x, t ; \ell) \ln P(x, t ; \ell) d x \\
& =\sum_{\ell} r(\ell) \int P(x, t ; \ell) \ln \frac{P(x, t ; \ell)}{P^{\prime}(x ; \ell)} d x-\sum_{\ell} r(\ell) \ln z_{P^{\prime}}(\ell),
\end{aligned}
$$

where the first term is a non-negative Kullback-Leibler divergence of $P^{\prime}(x ; \ell)$ from $P(x, t ; \ell)[7,32,46,52]$. From this equation, $I[P(x, t ; k), Q(x ; k)] \geqslant-\sum_{\ell} r(\ell) \ln z_{P}(\ell)>-\infty$ follows.

[1] A. M. Turing, Philos. Trans. R. Soc. London, Ser. B 237, 37 (1952).

[2] P. S. Hagan, SIAM J. Appl. Math. 42, 762 (1982).

[3] M. C. Cross and P. C. Hohenberg, Rev. Mod. Phys. 65, 851 (1993).

[4] J. A. Acebrón, L. L. Bonilla, C. J. Pérez Vicente, F. Ritort, and R. Spigler, Rev. Mod. Phys. 77, 137 (2005).

[5] R. C. Desai and R. Zwanzig, J. Stat. Phys. 19, 1 (1978).

[6] D. A. Dawson, J. Stat. Phys. 31, 29 (1983).

[7] M. Shiino, Phys. Rev. A 36, 2393 (1987).

[8] J. García-Ojalvo and J. M. Sancho, Noise in Spatially Extended Systems (Springer, New York, 1999).

[9] N. Goldenfeld, Lectures on Phase Transitions and the Renormalization Group (Addison-Wesley, Reading, MA, 1992).

[10] K. Kawasaki and T. Ohta, Physica A 116, 573 (1982).

[11] D. H. Zanette, Phys. Rev. E 55, 5315 (1997).

[12] P. G. Lind, J. Corte-Real, and J. A. C. Gallas, Phys. Rev. E 69, 026209 (2004)

[13] B. Ermentrout, X. Chen, and Z. Chen, Physica D 108, 147 (1997).

[14] A. R. Bulsara and G. Schmera, Phys. Rev. E 47, 3734 (1993).

[15] J. M. Casado, J. Gómez-Ordóñez, and M. Morillo, Europhys. Lett. 79, 50002 (2007).

[16] A. S. Pikovsky, A. Zaikin, and M. A. de la Casa, Phys. Rev. Lett. 88, 050601 (2002).

[17] C. J. Tessone, C. R. Mirasso, R. Toral, and J. D. Gunton, Phys. Rev. Lett. 97, 194101 (2006).

[18] R. Toral, C. Mirasso, and J. Gunton, Europhys. Lett. 61, 162 (2003).

[19] H. Nakao and A. S. Mikhailov, Phys. Rev. E 79, 036214 (2009).

[20] T. Ichinomiya, Phys. Rev. E 70, 026116 (2004).

[21] J. G. Restrepo, E. Ott, and B. R. Hunt, Phys. Rev. E 71, 036151 (2005).

[22] T. W. Ko and G. B. Ermentrout, Phys. Rev. E 78, 016203 (2008).

[23] H. Nakao and A. S. Mikhailov, Nat. Phys. 6, 544 (2010).

[24] S. Hata, H. Nakao, and A. S. Mikhailov, Europhys. Lett. 98, 64004 (2012).

[25] M. Wolfrum, Physica D 241, 1351 (2012).

[26] C. N. Angstmann, I. C. Donnelly, and B. I. Henry, Phys. Rev. E 87, 032804 (2013).
[27] N. E. Kouvaris, H. Kori, and A. S. Mikhailov, PLoS ONE 7, e45029 (2012).

[28] N. E. Kouvaris and A. S. Mikhailov, Europhys. Lett. 102, 16003 (2013).

[29] J. A. Acebrón, S. Lozano, and A. Arenas, Phys. Rev. Lett. 99, 128701 (2007).

[30] M. Perc, Phys. Rev. E 78, 036105 (2008).

[31] M. Perc and M. Gosak, New J. Phys. 10, 053008 (2008).

[32] T. Frank, A. Daffertshofer, C. Peper, P. Beek, and H. Haken, Physica D 150, 219 (2001).

[33] T. Frank and A. Daffertshofer, Physica A 295, 455 (2001).

[34] R. Albert and A.-L. Barabási, Rev. Mod. Phys. 74, 47 (2002).

[35] M. E. J. Newman, S. H. Strogatz, and D. J. Watts, Phys. Rev. E 64, 026118 (2001)

[36] The exponent $\gamma=2.5$ has no particular meaning in this study. When the parameters $\epsilon, k_{\min }, k_{\max }$, and $D$ are specified, we observe qualitatively the same dynamics for $2<\gamma \leqslant 3$, though the total relaxation time of the system may slightly vary.

[37] R. Lambiotte, M. Ausloos, and J. A. Hołyst, Phys. Rev. E 75, 030101 (2007).

[38] D. Bray, J. Theor. Biol. 143, 215 (1990).

[39] N. Barkai and S. Leibler, Nature 387, 913 (1997).

[40] B. Andò, S. Baglio, A. R. Bulsara, and V. Sacco, IEEE Inst. Meas. Mag. 8, 64 (2005).

[41] R. Pastor-Satorras and A. Vespignani, Phys. Rev. Lett. 86, 3200 (2001).

[42] A. Barrat, M. Barthélemy, and A. Vespignani, Dynamical Processes on Complex Networks (Cambridge University Press, Cambridge, 2008).

[43] It is known that the networks generated by the configuration model possess a certain level of degree correlation. We confirmed that this correlation is sufficiently weak and the meanfield approximation works nicely by comparing the numerical results obtained by using the standard configuration model with those obtained by using the uncorrelated configuration model [51].

[44] H. A. Kramers, Physica 7, 284 (1940).

[45] R. Ferrando, R. Spadacini, and G. E. Tommei, Phys. Rev. E 48, 2437 (1993).

[46] H. Risken, The Fokker-Planck Equation: Methods of Solution and Applications (Springer, Berlin, 1989). 
[47] C. W. Gardiner, Stochastic Methods: A Handbookfor the Natural and Social Sciences (Springer, New York, 2009).

[48] K. Sznajd-Weron and J. Sznajd, Int. J. Mod. Phys. C 11, 1157 (2000).

[49] A. T. Bernardes, D. Stauffer, and J. Kertész, Eur. Phys. J. B 25 , 123 (2002).
[50] P. Nyczka, J. Cisło, and K. Sznajd-Weron, Physica A 391, 317 (2012).

[51] M. Catanzaro, M. Boguñá, and R. Pastor-Satorras, Phys. Rev. E 71, 027103 (2005).

[52] S. Kullback and R. A. Leibler, Ann. Math. Stat. 22, 79 (1951). 